\title{
La prospection des ligneux fourragers dans la Communauté économique des Pays des Grands Lacs (Burundi, Rwanda, Zaïre)
}

\author{
Muzinga Kanzila ${ }^{1}$
}

MUZINGA KANZILA. La prospection des ligneux fourragers dans la Communauté économique des Pays des Grands Lacs (Burundi, Rwanda, Zaïre). Revue Élev. Méd. vét. Pays trop., 1994, 47 (4) : 415-424

Les prospections faites auprès des éleveurs et bouviers des grandes régions d'élevage du Burundi, du Rwanda et du Zaïre, ont permis d'inventorier 242 espèces d'arbres et arbustes indigènes consommés par les bovins, caprins et ovins. Ces espèces sont réparties dans 53 familles botaniques. Tous les ruminants domestiques des régions prospectées s'adonnent au brout à l'une ou l'autre époque de l'année, selon la disponibilité en herbes fourragères et surtout selon l'espèce animale à laquelle ils appartiennent. L'époque de prélèvement maximal reste la saison sèche. $L$ 'éleveur traditionnel a une bonne connaissance des arbres et arbustes fourragers indigènes mais les principaux usages qu'il leur reconnaît sont ceux de tout arbre. L'usage fourrager est simplement observé, ce qui explique que les arbres et arbustes fourragers ne font pas l'objet d'un traitement particulier. L'analyse chimique de quelques espèces a été effectuée et s'est révélée intéressante avec une moyenne en matières azotées de 18,5 p. 100 de la matière sèche en saison des pluies et 17,6 p. 100 en saison sèche.

Mots clés : Bovin - Ovin - Caprin - Plante de brout - Alimentation - Propriété organoleptique - Composition chimique - Saison - Burundi - Rwanda - Zäre

\section{INTRODUCTION}

Dans la Communauté économique des Pays des Grands Lacs comprenant le Burundi, le Rwanda et le Zaïre, l'alimentation des ruminants n'est souvent vue que sous l'angle de l'herbe. Or, l'herbe connaît deux contraintes majeures dans certaines parties de la communauté, à savoir : la longue saison sèche qui lui enlève l'essentiel de sa valeur alimentaire et l'exiguïté des terres destinées au pâturage herbeux, conséquence directe d'une démographie galopante.

Les arbres, arbustes et arbrisseaux peuvent contribuer à pallier cette carence dans la mesure où les feuilles et les fruits constituent des aliments pour le bétail. Certains restent verts durant toute l'année et leur composition chimique varie peu au cours des saisons. Leur rôle multiple leur permet de contribuer à l'amélioration des petites exploitations.

Quelques actions, basées essentiellement sur l'introduction, l'adaptation et l'évaluation de la production fourragère, sont entreprises dans le domaine des ligneux fourra-

1. Institut de Recherche agronomique et zootechnique (IRAZ) de la Communauté économique des Pays des Grands Lacs, BP 91, Gitega, Burundi.

Reçu le 17.6.1991, accepté le 14.2.1995. gers par les diverses institutions nationales mais celles-ci n'ont pas ou ont peu orienté leurs investigations sur la flore naturelle de la Communauté. Les espèces autochtones, qui ont le mérite de pousser dans leurs propres conditions écoclimatiques, seraient probablement plus faciles à vulgariser parce que déjà connues du paysan. L'étude des caractéristiques fourragères de ces arbres et arbustes indigènes passe d'abord par leur inventaire. Les prospections qui ont été menées pour ce faire ont visé à chercher dans la flore naturelle des essences susceptibles d'être utilisées en alimentation animale et de préférence dans un système agro-sylvo-pastoral. Cet inventaire non exhaustif donne une idée sur les potentialités en alimentation animale de la flore ligneuse des pays concernés. II constitue également un premier pas vers une étude plus approfondie de l'intérêt fourrager des espèces autochtones inventoriées.

\section{MATÉRIELS ET MÉTHODES}

Des prospections ont été menées sur les ligneux les plus courants par l'Institut de Recherche agronomique et zootechnique (IRAZ) de 1985 à 1989 dans les trois pays de la Communauté (carte 1), avec la participation des institutions nationales de recherche agronomique et de la plupart des projets de développement. Les herbiers nationaux y ont participé pour la détermination botanique des espèces. Ces prospections ont concerné les grandes régions écologiques d'élevage et ont consisté à entrer en contact avec un groupe d'éleveurs ou de gardiens et à parcourir avec eux les lieux où l'on trouve des essences fourragères naturelles. Pour chaque essence désignée, une fiche a été remplie sur la base des éléments suivants :

- nom vernaculaire et synonymes éventuels ;

- port et hauteur à l'âge adulte ;

- organes appétés (feuilles, fruits, écorces...) ;

- animaux concernés ;

- époque de consommation ;

- commentaires éventuels sur les particularités de l'espèce (degré d'appétibilité, mode de reproduction et autres usages).

Des questions générales complémentaires ont consisté à savoir si ces ligneux fourragers font l'objet d'une protection contre le feu et la coupe excessive, et s'ils sont 


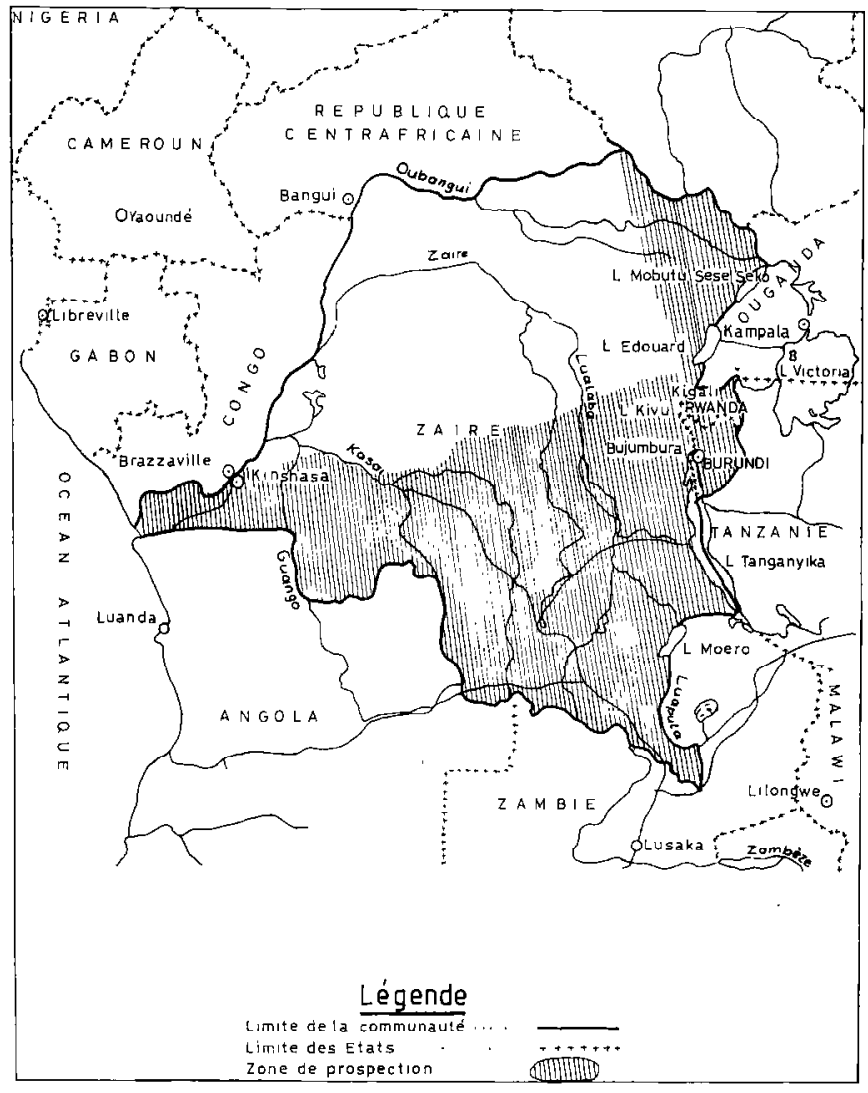

Carte 1 : Zone de prospection.

mis, d'une façon ou d'une autre, à la disposition des animaux par l'éleveur. Une fois ces éléments notés, un herbier a été récolté en vue d'une détermination botanique.

Les matières azotées totales ont été déterminées par la méthode de Kjeldhal, la cellulose par la méthode de Kurschner* modifiée, les cendres par incinération au four à mouffle à $450^{\circ} \mathrm{C}$ et les minéraux par absorption atomique : minéralisation avec $\mathrm{NHO}_{3}$ concentré après calcination.

\section{RÉSULTATS (tableau I)}

\section{Nombre et répartition botanique}

II a été inventorié 242 espèces d'arbres et arbustes dont certains organes sont consommés par des ruminants domestiques. Ces espèces sont réparties dans 53 familles botaniques dont les plus importantes, par le nombre d'espèces, sont Euphorbiaceae (24), Moraceae (24) Rubiaceae (21), Fabaceae (20), Mimosaceae (19), Caesalpiniaceae (13) et Verbenaceae (11).

*NDLR : cité par CHARLET-LERY, FRANCOIS A., LEROY A.M. (1952). L'analyse des aliments destinés aux animaux et l'interprétation des résultats qu'elle fournit. Annls Zootech., $3: 45-61$.

\section{Répartition régionale}

Les prospections au niveau national sont de 60 espèces au Burundi, 78 au Rwanda et 186 au Zaïre. On dénombre 24 espèces communes aux trois pays, 28 à deux pays, les autres étant limitées à un seul pays à la fois.

\section{Organes consommés}

Toutes les espèces prospectées sont surtout recherchées pour leurs feuilles et extrémités de tiges (98 p. 100) mais aussi pour leurs fruits (23 p. 100).

\section{Animaux consommateurs}

Tous les ruminants domestiques des régions prospectées recourent au brout, l'intensité dépendant de l'espèce animale. Les animaux cueillent eux-mêmes les organes qui les intéressent dans la mesure où ceux-ci leur sont accessibles.

\section{Saison de consommation}

Fait inhabituel et encore inexpliqué, il ressort des prospections que la plupart des espèces sont broutées indistinctement en toutes saisons (84 p. 100). II faut cependant constater que l'intensité est un peu plus prononcée en saison sèche ( 97 p. 100 contre 86 p. 100 en saison des pluies).

\section{Degré d'appétibilité}

L'information contenue dans le tableau de synthèse sur ce critère est générale et indicative. Il convenait d'apprendre de l'éleveur le comportement des animaux vis-à-vis de chaque espèce :

- très : quand l'espèce est recherchée par les animaux au point d'en manger presque à satiété ;

- moyen : quand l'espèce est broutée en complément des herbacées:

- peu : quand l'espèce est broutée occasionnellement parce que l'animal la trouve sur son passage.

Ces informations qualitatives pourraient être complétées par des tests d'appétibilité relative ${ }^{* *}$ et des mesures de consommations en stabulation.

\section{Mode de multiplication}

Dans leur grande majorité, les espèces prospectées peuvent se reproduire par les graines mais aussi par voie végétative. Ces informations sont indicatives car l'éleveur n'agit pas directement sur la multiplication, les graines germant naturellement ou après passage par le tube digestif d'oiseaux ou de ruminants. Les piquets de clôture ou de construction peuvent aussi donner lieu à une forme de bouturage inattendu.

\footnotetext{
** Comparaison des ordres, priorités d'ingestion de plusieurs espèces
} fourragères proposées simultanément à un groupe d'animaux. 
TABLEAU I Synthèse des résultats des prospections des arbres et arbustes fourragers naturels dans les pays de la CEPGL (Burundi, Rwanda, Zaïre).

\begin{tabular}{|c|c|c|c|c|c|c|c|c|}
\hline $\begin{array}{l}\text { FAMILLE } \\
\text { Espèces }\end{array}$ & Pays & Port & $\begin{array}{l}\text { Organes } \\
\text { appétés }\end{array}$ & $\begin{array}{c}\text { Animaux } \\
\text { consomm. }\end{array}$ & Epoques & $\begin{array}{l}\text { Appéti- } \\
\text { bilité }\end{array}$ & $\begin{array}{l}\text { Multipli- } \\
\text { cation }\end{array}$ & $\begin{array}{c}\text { Autres } \\
\text { Usages }\end{array}$ \\
\hline \multicolumn{9}{|l|}{ ACANTHACEAE } \\
\hline Acanthus pubescens & $B, R, Z$ & a & $\mathrm{Fe}, \mathrm{Et}$ & $\mathrm{Bo}, \mathrm{Ca}, \mathrm{Ov}$ & SS,SP & $\mathrm{T}(\mathrm{Ca})$ & $V, G, R$ & - \\
\hline Brillantaisia cicatricosa & $B, R, Z$ & $\mathbf{s}$ & $\mathrm{Fe}, \mathrm{Et}$ & Bo, $\mathrm{Ca}, \mathrm{Ov}$ & SS,SP & - & - & - \\
\hline Mimulopsis arborescens & $\mathrm{R}$ & $a$ & $\mathrm{Fe}, \mathrm{FI}$ & $\mathrm{Bo}, \mathrm{Ca}, \mathrm{Ov}$ & SS,SP & - & $\ldots$ & - \\
\hline Mimulopsis solmsii & $\mathbf{R}$ & $b$ & $\mathrm{Fe}, \mathrm{Et}, \mathrm{FI}$ & $\mathrm{Bo}, \mathrm{Ca}, \mathrm{Ov}$ & SS,SP & - & - & - \\
\hline \multicolumn{9}{|l|}{ AGAVACEAE } \\
\hline Dracaena afromontana & $B, R, Z$ & a & $\mathrm{Fe}$ & $\mathrm{Bo}, \mathrm{Ca}, \mathrm{Ov}$ & SS,SP & $\mathrm{T}(\mathrm{Ca})$ & V,R & $\mathrm{C}, \mathrm{O}$ \\
\hline Dracaena nitens & Z & $a b, a$ & $\mathrm{Fe}$ & $\mathrm{Bo}, \mathrm{Ca}$ & SS,SP & $T$ & $\mathrm{C}, \mathrm{V}, \mathrm{R}$ & Méd \\
\hline \multicolumn{9}{|l|}{ ALANGIACEAE } \\
\hline Alangium chinense & Z & A & $\mathrm{Fe}$ & $\mathrm{Ca}, \mathrm{Ov}$ & SS,SP & - & $\mathrm{R}$ & - \\
\hline \multicolumn{9}{|l|}{ ANACARDIACEAE } \\
\hline Lannea antiscorbutica & Z & A & $\mathrm{JFe}$ & $\mathrm{Bo}, \mathrm{Ca}$ & SS,SP & $T$ & $\mathrm{~V}, \mathrm{R}$ & F,C,Méd \\
\hline Lannea welwitschii & Z & $A$ & $\mathrm{Fe}$ & $\mathrm{Bo}, \mathrm{Ca}, \mathrm{Ov}$ & SS,SP & $P$ à $T$ & $G, V, R$ & F,C,Méd \\
\hline Ozoroa reticulata & $\bar{R}$ & $\mathrm{a}$ à $\mathrm{A}$ & $\mathrm{Fe}, \mathrm{Et}$ & $\mathrm{Bo}, \mathrm{Ca}, \mathrm{Ov}$ & SS,SP & - & - & - \\
\hline Rhus longipes & $B, R, Z$ & $\mathrm{a}$ à $\mathrm{A}$ & $\mathrm{Fe}, \mathrm{Et}, \mathrm{Fr}$ & $\mathrm{Bo}, \mathrm{Ca}, \mathrm{Ov}$ & SS,SP & $\mathrm{T}$ & $G, R$ & F,Méd \\
\hline Rhus natalensis & $\mathrm{B}, \mathrm{R}, \mathrm{Z}$ & a & $\mathrm{Fe}, \mathrm{Et}, \mathrm{Fr}$ & $\mathrm{Bo}(\mathrm{SS}), \mathrm{Ca}, \mathrm{Ov}$ & SS,SP & $\mathrm{T}$ & $G, R$ & F,C,Méd \\
\hline Rhus vulgaris & $B, R, Z$ & $a$ & $\mathrm{Fe}, \mathrm{Et}, \mathrm{Fr}$ & $\mathrm{Bo}, \mathrm{Ca}, \mathrm{Ov}$ & SS,SP & $\mathrm{T}$ & $G, R$ & F,Méd \\
\hline Spondias mombin & Z & a à $A$ & $\mathrm{Fe}, \mathrm{Et}, \mathrm{Fr}$ & $\mathrm{Bo}, \mathrm{Ca}, \mathrm{Ov}$ & SS,SP & $\mathrm{T}$ & $G, V, R$ & F,C,Méd \\
\hline \multicolumn{9}{|l|}{ ANNONACEAE } \\
\hline Annona senegalensis & Z & a à $A$ & $\mathrm{JFe}, \mathrm{Fr}$ & Bo, $\mathrm{Ca}, \mathrm{Ov}$ & SS,SP & $M$ à $T$ & $V, G, R$ & F,Méd \\
\hline \multicolumn{9}{|l|}{ APOCYNACEAE } \\
\hline Carissa edulis & $\mathbf{R}$ & a & $\mathrm{Fe}, \mathrm{Et}, \mathrm{Fr}$ & $\mathrm{Bo}(\mathrm{Fr}), \mathrm{Ca}, \mathrm{Ov}$ & SS,SP & - & $G, R$ & - \\
\hline Diplorynchus condylocarpon & Z & $A$ & $\mathrm{Fe}, \mathrm{Fr}$ & $\mathrm{Bo}, \mathrm{Ca}, \mathrm{Ov}, \mathrm{Rs}$ & SS,SP & $\mathrm{P}$ à $\mathrm{T}$ & $\mathrm{V}, \mathrm{G}, \mathrm{R}$ & F,C,D,Méd \\
\hline \multicolumn{9}{|l|}{ ARALIACEAE } \\
\hline Cussonia arborea & $\mathbf{R}$ & a à $A$ & $\mathrm{Fe}, \mathrm{Et}$ & $\mathrm{Bo}, \mathrm{Ca}, \mathrm{Ov}$ & SS,SP & $M$ à $T$ & $V, R$ & $\mathrm{~F}, \mathrm{C}$ \\
\hline \multicolumn{9}{|l|}{ ASTERACEAE } \\
\hline Bothriocline longipes & $\mathrm{R}, \mathbf{Z}$ & a & $\mathrm{Fe}, \mathrm{Et}$ & $\mathrm{Bo}(\mathrm{SS}), \mathrm{Ca}, \mathrm{Ov}$ & SS,SP & $\mathrm{T}$ & $V, G, R$ & - \\
\hline Senecio mannii & $B, R, Z$ & a & $\mathrm{Fe}, \mathrm{Et}, \mathrm{Ec}$ & $\mathrm{Bo}, \mathrm{Ca}, \mathrm{Ov}$ & SS,SP & - & $G, R$ & - \\
\hline Senecio marangwensis & $R$ & $s$ & Fe,Et & $\mathrm{Bo}, \mathrm{Ca}, \mathrm{Ov}$ & SS,SP & - & - & - \\
\hline Vernonia adoensis & $\mathbf{R}$ & $\mathrm{b}$ à $\mathrm{a}$ & Fe,Et & $\mathrm{Bo}, \mathrm{Ca}, \mathrm{Ov}$ & SS,SP & - & $\mathrm{R}$ & Méd \\
\hline Vernonia amygdalina & $B, R, Z$ & $\mathrm{a}$ à $\mathrm{A}$ & $\mathrm{Fe}, \mathrm{Et}, \mathrm{Ec}$ & $\mathrm{Bo}, \mathrm{Ca}, \mathrm{Ov}$ & SS,SP & $\mathrm{P}$ à $\mathrm{T}$ & $\mathrm{G}, \mathrm{R}$ & F,C,Méd \\
\hline Vernonia auriculifera & $\mathrm{R}, \mathrm{Z}$ & a & $\mathrm{Fe}, \mathrm{Et}$ & $\mathrm{Bo}, \mathrm{Ca}, \mathrm{Ov}$ & SS,SP & - & $\mathrm{G}, \mathrm{R}$ & - \\
\hline Vernonia brachycalyx & $\mathrm{R}$ & $\mathrm{a}$ & $\mathrm{Fe}, \mathrm{Et}$ & $\mathrm{Bo}, \mathrm{Ca}, \mathrm{Ov}$ & SS,SP & - & - & - \\
\hline Vernonia hochstetteri & $R, Z$ & a & $\mathrm{Fe}, \mathrm{Et}$ & $\mathrm{Bo}, \mathrm{Ca}, \mathrm{OV}$ & SP & - & V & $\mathrm{F}$ \\
\hline Vernonia lasiopus & $B, R, Z$ & a & $\mathrm{Fe}, \mathrm{Et}$ & $\mathrm{Bo}, \mathrm{Ca}, \mathrm{Ov}$ & SS,SP & - & $\mathbf{R}$ & F,Méd \\
\hline \multicolumn{9}{|l|}{ BALANITACEAE } \\
\hline Balanites aegyptiaca & Z & A & $\mathrm{Fe}, \mathrm{Et}, \mathrm{Fr}$ & Bo, $\mathrm{Ca}, \mathrm{Ov}$ & SS,SP & $T$ & $\mathrm{G}, \mathrm{R}$ & $F, C, D$ \\
\hline \multicolumn{9}{|l|}{ BIGNONIACEAE } \\
\hline Kigelia africana & Z & a & $\mathrm{Fe}$ & $\mathrm{Bo}, \mathrm{Ca}, \mathrm{Ov}$ & SS,SP & $T$ & $V, G, R$ & F,C,Méd \\
\hline Markhamia lutea & $\bar{Z}$ & $\vec{A}$ & $\mathrm{Fe}$ & $\mathrm{Bo}, \mathrm{Ca}, \mathrm{Ov}$ & SS,SP & - & $\mathrm{R}$ & $F, A$ \\
\hline Spathodea campanulata & Z & A & $\mathrm{Fe}$ & Bo & SS,SP & $T$ & $G, R$ & F,C,A,Méd \\
\hline \multicolumn{9}{|l|}{ BURSERACEAE } \\
\hline Commiphora africana & $\mathrm{R}$ & a & $\mathrm{Fe}, \mathrm{Et}$ & $\mathrm{Ca}, \mathrm{Ov}$ & SS,SP & - & - & - \\
\hline Dacryodes edulis & Z & A & $\mathrm{Fe}, \mathrm{Fr}$ & $\mathrm{Ca}, \mathrm{Ov}$ & SS,SP & $\mathrm{T}$ & $\mathrm{G}, \mathrm{R}$ & $F, D$ \\
\hline \multicolumn{9}{|l|}{ CAESALPINIACEAE } \\
\hline Brachystegia boehmii & $\mathbf{Z}$ & $A$ & $\mathrm{JFe}$ & Bo & SS,SP & $T$ & $V, G, R$ & $\mathrm{C}$ \\
\hline Brachystegia spiciformis & Z & A & $\mathrm{JFe}$ & Bo, $\mathrm{Ca}, \mathrm{Rs}$ & SS & M & $\mathrm{R}$ & $\mathrm{F}, \mathrm{D}$ \\
\hline Brachystegia wangermeeana & Z & A & $\mathrm{JFe}$ & $\mathrm{Bo}, \mathrm{Ca}, \mathrm{Ov}$ & SS,SP & M & $G, R$ & $F, C, D$ \\
\hline Cassia didymobotrya & $\mathbf{R}$ & a & $\mathrm{Fe}, \mathrm{Fr}$ & $\mathrm{Bo}(\mathrm{SS}), \mathrm{Ca}, \mathrm{Ov}$ & SS,SP & - & - & - \\
\hline Cassia siamea & Z & A & $\mathrm{Fe}$ & Bo & SS & $\mathrm{T}$ & $V, G, R$ & $F, C$ \\
\hline
\end{tabular}


Muzinga Kanzila

TABLEAUI (suite)

\begin{tabular}{|c|c|c|c|c|c|c|c|c|}
\hline $\begin{array}{l}\text { FAMILLE } \\
\text { Espèces }\end{array}$ & Pays & Port & $\begin{array}{l}\text { Organes } \\
\text { appétés }\end{array}$ & $\begin{array}{l}\text { Animaux } \\
\text { consomm. }\end{array}$ & Epoques & $\begin{array}{l}\text { Appéti- } \\
\text { bilité }\end{array}$ & $\begin{array}{l}\text { Multipli- } \\
\text { cation }\end{array}$ & $\begin{array}{l}\text { Autres } \\
\text { Usages }\end{array}$ \\
\hline Cassia spectabilis & $\mathbf{Z}$ & a à $A$ & JFe,Go & Bo & SS,SP & $\mathrm{T}(\mathrm{Go}) \mathrm{P}(\mathrm{Go})$ & $V, G, R$ & $\mathrm{~F}, \mathrm{C}$ \\
\hline Daniellia alsteeniana & Z & $A$ & JFe,FI & $\mathrm{Bo}, \mathrm{Ca}$ & SS,SP & $\mathrm{T}$ & $V, G, R$ & F,C,A,Méd \\
\hline Dialium englerianum & Z & A & $\mathrm{Fe}, \mathrm{Fr}$ & $\mathrm{Bo}, \mathrm{Ca}$ & SS,SP & $\mathrm{T}$ & $\mathrm{G}, \mathrm{R}$ & F,Méd \\
\hline Julbernardia paniculata & Z & A & $\mathrm{Fe}$ & Bo,Rs & SS,SP & $M$ & $G, R$ & $\mathrm{~F}, \mathrm{C}$ \\
\hline Pauletia tomentosa & $\mathbf{Z}$ & $a$ & $\mathrm{Fe}$ & $\mathrm{Ca}, \mathrm{OV}$ & SS,SP & $\mathrm{T}$ & $\mathrm{G}, \mathrm{R}$ & $\mathrm{F}, \mathrm{C}$ \\
\hline Piliostigma thonningii & Z & $a$ à $A$ & $\mathrm{JFe}, \mathrm{Go}$ & $\mathrm{Bo}, \mathrm{Ca}, \mathrm{Ov}, \mathrm{Rs}$ & SS,SP & $P$ à $T$ & $\mathrm{G}, \mathrm{R}$ & F,C,Méd \\
\hline Swartzia madagascariensis & Z & a à $A$ & JFe,Go & $\mathrm{Bo}, \mathrm{Ca}, \mathrm{Ov}$ & SS,SP & $\mathrm{T}(\mathrm{Go}) \mathrm{M}(\mathrm{Fe})$ & G,R & F,C,D,Méd \\
\hline Tamarindus indica & Z & A & $\mathrm{Fe}, \mathrm{Et}, \mathrm{Go}$ & $\mathrm{Bo}, \mathrm{Ca}, \mathrm{Ov}$ & SS & $T(G o)$ & $\mathrm{G}, \mathrm{R}$ & $F, C$ \\
\hline \multicolumn{9}{|l|}{ CAPPARACEAE } \\
\hline Capparis erythrocarpos & $\mathrm{R}$ & a & $\mathrm{Fe}, \mathrm{Et}, \mathrm{Fr}$ & $\mathrm{Ca}, \mathrm{Ov}$ & SS,SP & 一 & - & - \\
\hline $\begin{array}{c}\text { Capparis fascicularis } \\
\text { var. elaegnoides }\end{array}$ & $\mathrm{R}$ & a & $\mathrm{Fe}, \mathrm{Et}$ & Bo CaOv & SS SP & $\mathrm{T}(\mathrm{Ca}$ Ov) & - & - \\
\hline Maerua angolensis & $\mathrm{B}$ & a & $\mathrm{Fe}$ & $\begin{array}{l}\text { Bo, La, Uv } \\
\text { Bo }\end{array}$ & SS,SP & (ud, & - & - \\
\hline Maerua triphylla & $\mathrm{R}$ & a & $\mathrm{Fe}, \mathrm{Et}, \mathrm{Fl}, \mathrm{Fr}$ & $\mathrm{Bo}, \mathrm{Ca}, \mathrm{Ov}$ & SS,SP & - & - & - \\
\hline \multicolumn{9}{|l|}{ CELASTRACEAE } \\
\hline Maytenus acuminata & Z & a & $\mathrm{Fe}, \mathrm{Et}, \mathrm{Ec}$ & $\mathrm{Ca}, \mathrm{OV}$ & SS,SP & - & $\mathrm{R}$ & - \\
\hline Maytenus arbutifolia & $\mathrm{B}$ & a & $\mathrm{Fe}$ & $\mathrm{Ca}$ & SS,SP & - & - & - \\
\hline Maytenus heterophylla & $R, Z$ & a & $\mathrm{Fe}, \mathrm{Et}, \mathrm{FI}, \mathrm{Fr}$ & $\mathrm{Ca}, \mathrm{Ov}$ & SS,SP & - & - & - \\
\hline Maytenus sp. & Z & A & $\mathrm{Fe}, \mathrm{Fr}$ & Bo,Rs & SS,SP & M & $G, R$ & F,C,Méd \\
\hline \multicolumn{9}{|l|}{ CHRYSOBALANACEAE } \\
\hline Parinari capensis & Z & $a b$ & $\mathrm{JFe}$ & Bo & SS & $\mathrm{P}$ & $\mathrm{V}, \mathrm{R}$ & F,Méd \\
\hline Parinari curatellifolia & $B, R$ & a & $\mathrm{Fe}, \mathrm{Et}$ & $\mathrm{Bo}, \mathrm{Ca}, \mathrm{OV}$ & SS,SP & $\mathrm{T}(\mathrm{SS})$ & $\mathrm{R}$ & F,Méd \\
\hline Parinari mobola & Z & A & $\mathrm{JFe}, \mathrm{Fr}$ & Bo, Ca,Ov, Rs & SS,SP & $T(F r) M(F e)$ & $G, R$ & F,C,D,Méd \\
\hline \multicolumn{9}{|l|}{ CLUSIACEAE } \\
\hline Harungana madagascariensis & $B, Z$ & a & $\mathrm{Fe}, \mathrm{Et}, \mathrm{Ec}$ & $\mathrm{Bo}, \mathrm{Ca}, \mathrm{Ov}$ & SS,SP & $T$ & $G, R$ & F,C,Méd \\
\hline Hypericum revolutum & $R, Z$ & $b$ & $\mathrm{Fe}, \mathrm{Et}, \mathrm{FI}$ & $\mathrm{Bo}, \mathrm{Ca}, \mathrm{Ov}$ & SS,SP & - & $V, R$ & D \\
\hline Psorospermum febrifugum & $B, R, Z$ & a & Fe,Et & $\mathrm{Bo}, \mathrm{Ca}, \mathrm{Ov}$ & SS,SP & $M$ à $T$ & $G, R$ & F,Méd \\
\hline Psorospermum ferrugineum & Z & a & $\mathrm{JFe}$ & $\mathrm{Ca}, \mathrm{OV}$ & SS,SP & $M$ & $\mathrm{G}, \mathrm{R}$ & Méd \\
\hline \multicolumn{9}{|l|}{ COMBRETACEAE } \\
\hline Combretum binderianum & Z & a & $\mathrm{Fe}$ & $\mathrm{Bo}, \mathrm{Ca}, \mathrm{Ov}$ & SS & $M$ & $G, R$ & F,C,Méd \\
\hline Combretum collinum & $\overline{\mathrm{R}}$ & $\mathrm{a}$ à $\mathrm{A}$ & $\mathrm{Fe}, \mathrm{JFr}$ & $\mathrm{Bo}, \mathrm{Ca}, \mathrm{Ov}$ & SS,SP & - & - & - \\
\hline Combretum ryheri & $Z$ & $a$ & Ec & $\mathrm{Bo}, \mathrm{Ca}$ & SS,SP & $M$ & V & $F$ \\
\hline Terminalia superba & Z & A & $\mathrm{Fe}, \mathrm{Et}, \mathrm{JPI}$ & Bo & SS,SP & $\mathrm{T}$ & $\mathrm{G}, \mathrm{R}$ & F,I,Méd \\
\hline \multicolumn{9}{|l|}{ DILLENIACEAE } \\
\hline Tetracera poggei & $z$ & $a b$ & $\mathrm{Fe}$ & $\mathrm{Bo}, \mathrm{Ca}, \mathrm{Ov}$ & SS,SP & M & $G, R$ & Méd \\
\hline \multicolumn{9}{|l|}{ EBENACEAE } \\
\hline Euclea schimperi & $\mathrm{R}$ & a & $\mathrm{Fe}, \mathrm{Et}$ & $\mathrm{Bo}, \mathrm{Ca}, \mathrm{Ov}$ & SS,SP & - & $\mathrm{R}$ & - \\
\hline \multicolumn{9}{|l|}{ ERICACEAE } \\
\hline Phillipia benguellensis & z & a & $\mathrm{Fe}, \mathrm{Et}, \mathrm{FI}$ & $\mathrm{Bo}, \mathrm{Ca}, \mathrm{Ov}$ & SS & 一 & - & $\mathrm{F}$ \\
\hline \multicolumn{9}{|l|}{ EUPHORBIACEAE } \\
\hline Acalypha bipartita & $\mathrm{R}$ & $\mathrm{a}$ & $\mathrm{Fe}, \mathrm{Et}$ & $\mathrm{Ca}$ & SS,SP & - & - & - \\
\hline Alchornea cordifolia & $\mathrm{Z}$ & $\mathrm{a}$ à $\mathrm{A}$ & $\mathrm{JFe}$ & $\mathrm{Bo}, \mathrm{Ca}, \mathrm{Ov}, \mathrm{Rs}$ & SS,SP & $P$ à $T$ & $\mathrm{~V}, \mathrm{G}, \mathrm{R}$ & F,C,A,Méd \\
\hline Bridelia brideliifolia & $\bar{B}$ & A & $\mathrm{Fe}, \mathrm{Et}$ & $\mathrm{Bo}, \mathrm{Ca}, \mathrm{Ov}$ & SS,SP & - & $\mathrm{R}$ & $F, C$ \\
\hline Bridelia ferruginea & Z & $a$ à $A$ & $\mathrm{JFe}, \mathrm{Et}$ & $\mathrm{Bo}, \mathrm{Ca}, \mathrm{Ov}, \mathrm{Rs}$ & SS,SP & $P$ & $G, R$ & F,Méd \\
\hline Bridelia micrantha & Z & $\mathrm{a}$ à $\mathrm{A}$ & $\mathrm{Fe}$ & $\mathrm{Bo}, \mathrm{Ca}, \mathrm{Ov}$ & SS & M à T & $\mathrm{G}, \mathrm{R}$ & F,C,Méd \\
\hline Croton macrostachyus & $\mathrm{B}$ & A & $\mathrm{Fe}, \mathrm{Et}$ & $\mathrm{Bo}, \mathrm{Ca}, \mathrm{Ov}$ & SS,SP & $\mathrm{T}(\mathrm{Bo})$ & $\mathrm{R}$ & $F, C$ \\
\hline Erytrococca bongensis & $B, R, Z$ & a & $\mathrm{Fe}, \mathrm{Et}$ & $\mathrm{Bo}, \mathrm{Ca}, \mathrm{Ov}$ & SS,SP & - & $\mathrm{R}$ & $F$ \\
\hline Erytrococca trichogyne & $\mathrm{B}$ & a & $\mathrm{Fe}, \mathrm{Et}$ & $\mathrm{Bo}, \mathrm{Ca}, \mathrm{Ov}$ & SS,SP & $\mathrm{T}(\mathrm{Ca})$ & $\mathrm{R}$ & $\mathrm{F}, \mathrm{C}$ \\
\hline Euphorbia tirucalli & $B, R, Z$ & a à $A$ & Ti tendre & $\mathrm{Ca}, \mathrm{Ov}$ & SS,SP & $\mathrm{T}(\mathrm{Ca})$ & $\mathrm{V}, \mathrm{R}$ & C,Méd \\
\hline Euphorbiaceae sp. $n^{\circ} 1$ & Z & A & $\mathrm{Fe}, \mathrm{Ec}$ & $\mathrm{Ca}$ & SS,SP & $T$ & $\mathrm{G}$ & F,C,Méd \\
\hline Euphorbiaceae sp. $n^{\circ} 2$ & $\bar{z}$ & $a b$ & $\mathrm{Fe}, \mathrm{Et}$ & $\mathrm{Ca}, \mathrm{Ov}$ & SS & $P$ & $\mathrm{G}, \mathrm{R}$ & $\mathrm{F}$ \\
\hline Hymenocardia acida & & $\mathrm{a}$ à $\mathrm{A}$ & $\mathrm{JFe}, \mathrm{Et}, \mathrm{Ec}$ & $\mathrm{Bo}, \mathrm{Ca}, \mathrm{Ov}, \mathrm{Rs}$ & SS,SP & $\mathrm{P}$ à $\mathrm{T}$ & $\mathrm{G}, \mathrm{R}$ & F,C,Méd \\
\hline
\end{tabular}


TABLEAU I (suite)

\begin{tabular}{|c|c|c|c|c|c|c|c|c|}
\hline $\begin{array}{l}\text { FAMILLE } \\
\text { Espèces }\end{array}$ & Pays & Port & $\begin{array}{l}\text { Organes } \\
\text { appétés }\end{array}$ & $\begin{array}{c}\text { Animaux } \\
\text { consomm. }\end{array}$ & Epoques & $\begin{array}{l}\text { Appéti- } \\
\text { bilité }\end{array}$ & $\begin{array}{c}\text { Multipli- } \\
\text { cation }\end{array}$ & $\begin{array}{l}\text { Autres } \\
\text { Usages }\end{array}$ \\
\hline Hymenocardia ulmoides & Z & $A$ & $\mathrm{Fe}, \mathrm{Ec}$ & $\mathrm{Bo}, \mathrm{Ca}, \mathrm{Ov}$ & SS,SP & $\mathrm{T}(\mathrm{Ca}) \mathrm{P}(\mathrm{Bo})$ & $R$ & F,C,Méd \\
\hline Macaranga kilimandscharica & $\mathbf{Z}$ & $A$ & $\mathrm{Fe}, \mathrm{Et}$ & $\mathrm{Bo}, \mathrm{Ca}, \mathrm{Ov}$ & SS,SP & - & $\mathrm{R}$ & $F, C$ \\
\hline Maprounea africana & $\bar{Z}$ & $a$ & JFe & $\mathrm{Ca}, \mathrm{Ov}$ & SS,SP & $M$ à $T$ & $G, R$ & Méd \\
\hline Phyllanthus muellerianus & $z$ & a & $\mathrm{JFe}, \mathrm{Fr}$ & $\mathrm{Bo}, \mathrm{Ca}, \mathrm{Ov}, \mathrm{Rs}$ & SS,SP & $\mathrm{P}$ à $\mathrm{T}$ & $V, G, R$ & F,C,Méd \\
\hline Phyllanthus ovalifolius & $\mathrm{B}$ & a & $\mathrm{Fe}$ & $\mathrm{Bo}, \mathrm{Ca}$ & SS & - & - & Mèd \\
\hline Pseudolachnostylis & & & & & & & & \\
\hline maprouneifolia & Z & $\mathrm{a}$ à $\mathrm{A}$ & $\mathrm{JFe}, \mathrm{Fr}$ & $\mathrm{Bo}, \mathrm{Ca}, \mathrm{Ov}, \mathrm{Rs}$ & SS,SP & $P$ à $M$ & $V, G, R$ & F,C,Méd \\
\hline Sapium ellipticum & Z & a à $A$ & JFe,Ec & $\mathrm{Bo}, \mathrm{Ca}, \mathrm{Ov}$ & SS,SP & $M$ & $G, R$ & F,Méd \\
\hline Securinega virosa & $\mathrm{B}, \bar{Z}, \mathrm{R}$ & a & $\mathrm{Fe}, \mathrm{Et}$ & $\mathrm{Ca}$ & SS,SP & $\mathrm{M}$ à $\mathrm{T}$ & $V, G, R$ & F,C,Méd \\
\hline Synadenium grantii & $B, R$ & a & $\mathrm{Fe}$ & $\mathrm{Ca}, \mathrm{Ov}$ & SS & - & $\mathrm{V}, \mathrm{R}$ & - \\
\hline Uapaca guineensis & $Z$ & $A$ & $\mathrm{Fe}$ & $\mathrm{Ca}$ & SS,SP & M & $\mathrm{G}, \mathrm{R}$ & $\mathrm{F}, \mathrm{C}$ \\
\hline Uapaca pilosa & $\mathbf{Z}$ & $a$ & $\mathrm{JFe}$ & Bo & SS,SP & $\mathrm{T}$ & $\mathrm{V}(\mathrm{SP}), \mathrm{G}, \mathrm{R}$ & $F$ \\
\hline Uapaca robynsii & Z & A & $\mathrm{Fe}$ & Bo,Rs & SS,SP & M & $\mathrm{G}, \mathrm{R}$ & F,C,Méd \\
\hline FABACEAE & & & & & & & & \\
\hline Aeschynomene schimperi & $\mathrm{R}$ & a & $\mathrm{Fe}, \mathrm{Et}$ & $\mathrm{Bo}, \mathrm{Ca}, \mathrm{Ov}$ & SS,SP & - & - & - \\
\hline Crotalaria agatiflora & $\mathrm{R}$ & a & $\mathrm{Fe}, \mathrm{Et}$ & $\mathrm{Bo}, \mathrm{Ca}, \mathrm{OV}$ & SS,SP & - & - & - \\
\hline Erythrina abyssinica & $B, R, Z$ & $\mathrm{a}$ à $\mathrm{A}$ & $\mathrm{Fe}, \mathrm{Et}$ & $\mathrm{Bo}, \mathrm{Ca}, \mathrm{Ov}$ & SS,SP & M & $V, G, R$ & Méd \\
\hline Erythrina tomentosa & Z & a & $\mathrm{JFe}$ & Bo & SS & $\mathbf{P}$ & V,G,R & F,C,A,D \\
\hline Indigofera arrecta & $B, \vec{R}, Z$ & a & $\mathrm{Fe}, \mathrm{Et}$ & $\mathrm{Bo}, \mathrm{Ca}, \mathrm{Ov}$ & SS,SP & - & V & F,Méd \\
\hline Indigofera emarginella & $\mathrm{R}$ & a & $\mathrm{Fe}, \mathrm{Et}$ & $\mathrm{Bo}, \mathrm{Ca}, \mathrm{Ov}$ & SS,SP & - & V,R & Méd \\
\hline Kotschya africana & $\mathbf{Z}$ & a & $\mathrm{Fe}, \mathrm{Fr}$ & Bo & SS,SP & M & $\mathrm{G}$ & C \\
\hline Kotschya aeschynomenoides & $\bar{B}$ & $\mathrm{a}$ & $\mathrm{Fe}, \mathrm{Et}, \mathrm{Fr}$ & $\mathrm{Bo}, \mathrm{Ca}, \mathrm{Ov}$ & SS,SP & $\mathrm{T}(\mathrm{Ca})$ & - & $\mathrm{F}, \mathrm{C}$ \\
\hline Kotschya africana & $\mathrm{B}, \mathrm{Z}$ & $b$ & $\mathrm{Fe}, \mathrm{Et}, \mathrm{Fr}$ & $\mathrm{Bo}, \mathrm{Ca}, \mathrm{Ov}$ & SS,SP & $\mathrm{T}(\mathrm{Ca})$ & - & $\mathrm{F}, \mathrm{C}$ \\
\hline Kotschya strigosa & $\mathrm{B}$ & s & $\mathrm{Fe}, \mathrm{Et}, \mathrm{Fr}$ & $\mathrm{Bo}, \mathrm{Ca}, \mathrm{Ov}$ & SS,SP & $\mathrm{T}(\mathrm{Ca})$ & - & $\mathrm{F}, \mathrm{C}$ \\
\hline Leptoderris nobilis & Z & $a b$ & $\mathrm{JFe}$ & Bo & SS,SP & $\mathrm{T}$ & $R$ & Méd \\
\hline Milletia dura & $\mathrm{B}, \mathrm{Z}$ & a & $\mathrm{Fe}, \mathrm{Et}$ & $\mathrm{Bo}, \mathrm{Ca}, \mathrm{Ov}$ & SS,SP & - & $\mathrm{R}$ & F,C,Méd \\
\hline Milletia laurentii & $\mathbf{Z}$ & $A$ & $\mathrm{Fe}$ & $\mathrm{Ca}, \mathrm{Ov}$ & SS,SP & $M$ & $V, G, R$ & $\mathrm{~F}, \mathrm{C}$ \\
\hline Milletia versicolor & $Z$ & A & $\mathrm{JFe}$ & $\mathrm{Bo}, \mathrm{Ca}, \mathrm{OV}$ & SS,SP & $M$ à $T$ & $V, G, R$ & F, C, A,D, Méc \\
\hline Ormocarpum trichocarpum & $\mathrm{R}$ & a & $\mathrm{Fe}, \mathrm{Et}, \mathrm{FI}, \mathrm{Fr}$ & $\mathrm{Bo}, \mathrm{Ca}, \mathrm{Ov}$ & SS,SP & - & - & - \\
\hline Pericopsis angolensis & Z & A & $\mathrm{JFe}$ & $\mathrm{Bo}, \mathrm{Ca}$ & SS & $M$ & V & F,C,Méd \\
\hline Sesbania sesban & Z & a & $\mathrm{Fe}, \mathrm{Et}$ & $\mathrm{Bo}, \mathrm{Ca}, \mathrm{Ov}$ & SS,SP & $\mathrm{T}$ & $G, R$ & $F$ \\
\hline Sesbania sesban var. nubica & $\mathrm{R}, \mathrm{Z}$ & a & $\mathrm{Fe}, \mathrm{Et}$ & $\mathrm{Bo}, \mathrm{Ca}, \mathrm{Ov}$ & SS,SP & 一 & $\mathrm{G}, \mathrm{R}$ & C,Méd \\
\hline $\begin{array}{l}\text { var. mildbraedii } \\
\text { varosia mierrupta }\end{array}$ & Z & a & $\mathrm{Fe}, \mathrm{Et}$ & $\mathrm{Bo}, \mathrm{Ca}, \mathrm{Ov}$ & SS,SP & - & - & $\mathrm{F}$ \\
\hline Tephrosia vogelii & $R, Z$ & a & $\mathrm{Fe}, \mathrm{Et}$ & $\mathrm{Bo}, \mathrm{Ca}, \mathrm{Ov}$ & SS,SP & - & - & - \\
\hline FLACOURTIACEAE & & & & & & & & \\
\hline Caloncoba welwitschii & Z & a à $A$ & $\mathrm{Fe}, \mathrm{Et}$ & $\mathrm{Ca}, \mathrm{Ov}, \mathrm{Rs}$ & SS,SP & $M$ à $T$ & $\mathrm{G}, \mathrm{R}$ & F,C,Méd \\
\hline Lindackeria kivuensis & $\mathrm{B}$ & $\mathrm{a}$ & $\mathrm{Fe}, \mathrm{Et}$ & $\mathrm{Bo}, \mathrm{Ca}, \mathrm{Ov}$ & SS,SP & $\mathrm{T}(\mathrm{Bo})$ & $\mathrm{R}$ & F,C, Méd \\
\hline Paropsia brazzeana & $\mathbf{Z}$ & $a b$ & $\mathrm{JFe}$ & $\mathrm{Bo}, \mathrm{Ca}$ & SS,SP & $\mathrm{P}$ & V & $F$ \\
\hline HIPPOCRATEACEAE & & & & & & & & \\
\hline Loeseneriella apiculata & Z & $a b$ & $\mathrm{JFe}$ & $\mathrm{Bo}, \mathrm{Ca}, \mathrm{Ov}$ & SS,SP & $M$ & $\mathrm{G}, \mathrm{R}$ & F,A,Méd \\
\hline LAMIACEAE & & & & & & & & \\
\hline Hoslundia opposita & $z$ & B & $\mathrm{Fe}$ & $\mathrm{Bo}, \mathrm{Ca}, \mathrm{Ov}$ & SS,SP & $M$ & $G, R$ & - \\
\hline LOBELIACEAE & & & & & & & & \\
\hline Lobelia giberroa & $\mathrm{R}$ & $a b$ & $\mathrm{Fe}, \mathrm{Et}$ & $\mathrm{Bo}, \mathrm{Ca}, \mathrm{Ov}$ & SS,SP & - & - & - \\
\hline Lobelia mildbraedii & Z & $a b$ & $\mathrm{Fe}, \mathrm{Et}$ & $\mathrm{Bo}, \mathrm{Ca}, \mathrm{Ov}$ & SS,SP & - & $\mathrm{R}$ & - \\
\hline LOGANIACEAE & & & & & & & & \\
\hline Anthocleista schweinfurthii & $\mathrm{B}$ & A & $\mathrm{Fe}$ & Bo & sS & - & - & - \\
\hline Nuxia floribunda & Z & $a$ à $A$ & $\mathrm{Fe}, \mathrm{Et}$ & Bo & $\mathrm{SP}$ & - & - & - \\
\hline Strychnos spinosa & Z & $a$ & $\mathrm{Fe}$ & $\mathrm{Bo}, \mathrm{Ca}, \mathrm{Ov}$ & SS,SP & $\mathrm{T}$ & $G, R$ & F,C,Méd \\
\hline Strychnos cocculoides & Z & $\mathrm{a}$ à $\mathrm{A}$ & $\mathrm{JFe}, \mathrm{Fr}$ & Bo, Ca, Ov, Rs & SP,SS & $M$ à $T$ & $\mathrm{G}, \mathrm{R}$ & F,A,Méd \\
\hline Strychnos pungens & $Z$ & a & $\mathrm{Fe}$ & $\mathrm{Bo}, \mathrm{Ca}, \mathrm{Ov}$ & SS,SP & $\mathrm{T}$ & $\mathrm{G}, \mathrm{R}$ & Méd \\
\hline MELASTOMATACEAE & & & & & & & & \\
\hline Dissotis trothae & B & $a b$ à $a$ & $\mathrm{Fe}, \mathrm{Et}$ & $\mathrm{Bo}, \mathrm{Ca}$ & SS,SP & - & - & - \\
\hline MELIANTHACEAE & & & & & & & & \\
\hline Bersama abyssinica & Z & $a$ & $\mathrm{Fe}$ & $\mathrm{Bo}, \mathrm{Ov}$ & SS,SP & - & $\mathrm{R}$ & - \\
\hline
\end{tabular}


TABLEAU I (suite)

\begin{tabular}{|c|c|c|c|c|c|c|c|c|}
\hline $\begin{array}{l}\text { FAMILLE } \\
\text { Espèces }\end{array}$ & Pays & Port & $\begin{array}{l}\text { Organes } \\
\text { appétés }\end{array}$ & $\begin{array}{l}\text { Animaux } \\
\text { consomm. }\end{array}$ & Epoques & $\begin{array}{l}\text { Appéti- } \\
\text { bilité }\end{array}$ & $\begin{array}{c}\text { Multipli- } \\
\text { cation }\end{array}$ & $\begin{array}{l}\text { Autres } \\
\text { Usages }\end{array}$ \\
\hline \multicolumn{9}{|l|}{ MIMOSACEAE } \\
\hline \multicolumn{9}{|l|}{ Acacia abyssinica } \\
\hline subsp. calophylla & $\mathrm{R}$ & A & $\mathrm{Fe}, \mathrm{Go}$ & $\mathrm{Bo}(\mathrm{Go}), \mathrm{Ov}$ & SS,SP & 一 & - & 一 \\
\hline Acacia cf. van meelii & Z & $A$ & $\mathrm{Fe}$ & $\mathrm{Bo}, \mathrm{Ca}, \mathrm{OV}$ & SS,SP & $\mathrm{T}$ & $\mathrm{G}, \mathrm{R}$ & $\mathrm{F}$ \\
\hline Acacia gerrardii & $\mathrm{B}, \mathrm{R}, \mathrm{Z}$ & a à $A$ & $\mathrm{Fe}, \mathrm{Et}$ & $\mathrm{Bo}(\mathrm{B}), \mathrm{Ca}, \mathrm{OV}$ & SS,SP & - & - & - \\
\hline Acacia hockii & $\mathrm{R}, \mathrm{Z}$ & $\mathrm{a}$ & $\mathrm{Fe}, \mathrm{Fl}, \mathrm{Go}$ & $\mathrm{Bo}(\mathrm{R}), \mathrm{Ca}, \mathrm{Ov}$ & SS & $\mathrm{T}(\mathrm{Ca})$ & $G, R$ & F,Ca,Méd \\
\hline Acacia polyacantha & Z & A & $\mathrm{Fe}$ & $\mathrm{Bo}, \mathrm{Ca}, \mathrm{Ov}$ & SS,SP & $\mathrm{T}$ & $\mathrm{G}, \mathrm{R}$ & F,Méd \\
\hline \multicolumn{9}{|l|}{ Acacia polyacantha } \\
\hline ssp. campylacantha & $\mathrm{R}$ & A & $\mathrm{Fe}, \mathrm{Et}, \mathrm{Go}$ & $\mathrm{Bo}(\mathrm{Go}), \mathrm{Ca}, \mathrm{Ov}$ & SS,SP & - & - & $\because$ \\
\hline Acacia senegal & $\mathrm{R}$ & a à $A$ & $\mathrm{Fe}, \mathrm{Et}$ & $\mathrm{Ca}, \mathrm{Ov}$ & SS,SP & - & - & - \\
\hline Acacia sieberiana $n^{\circ} 1$ & Z & A & $\mathrm{Fe}, \mathrm{Et}, \mathrm{Go}$ & $\mathrm{Bo}, \mathrm{Ca}, \mathrm{Ov}$ & SS,SP & $M$ & $G, R$ & $\mathrm{~F}, \mathrm{C}$ \\
\hline Acacia sieberiana $n^{\circ} 2$ & $\mathrm{~B}, \mathrm{R}$ & a à $A$ & $\mathrm{Fe}, \mathrm{Et}, \mathrm{Go}$ & $\mathrm{Bo}, \mathrm{Ca}, \mathrm{Ov}$ & SS,SP & $\mathrm{T}(\mathrm{Bo}, \mathrm{SS})$ & - & - \\
\hline Acacia sp. & Z & a à $A$ & $\mathrm{Fe}, \mathrm{Et}, \mathrm{Go}$ & $\mathrm{Bo}, \mathrm{Ca}, \mathrm{Ov}$ & SS,SP & $\mathrm{M}$ à $\mathrm{T}$ & $G, R$ & F,Méd \\
\hline Albizzia adianthifolia & $B, R, Z$ & A & $\mathrm{Fe}, \mathrm{Et}, \mathrm{Ec}$ & $\mathrm{Bo}, \mathrm{Ca}, \mathrm{Ov}, \mathrm{Rs}$ & SS,SP & $M$ à $T$ & $V, G, R$ & $F, C, A$, Méd \\
\hline Albizzia antunesiana & Z & A & $\mathrm{Fe}, \mathrm{Et}$ & $\mathrm{Bo}, \mathrm{Ca}, \mathrm{OV}$ & SS,SP & $M$ à $T$ & $\mathrm{G}, \mathrm{R}$ & F,C,A,Méd \\
\hline Albizzia cf. grandibracteata & Z & A & $\mathrm{Fe}, \mathrm{Et}, \mathrm{Ec}$ & $\mathrm{Bo}, \mathrm{Ca}, \mathrm{Ov}$ & SS,SP & - & $\mathrm{R}$ & $\mathrm{F}, \mathrm{C}, \mathrm{A}$ \\
\hline Albizzia gummifera & Z & $\mathrm{a}$ à $\mathrm{A}$ & $\mathrm{Fe}, \mathrm{Et}$ & $\mathrm{Bo}, \mathrm{Ca}, \mathrm{Ov}$ & SS,SP & $\mathrm{T}$ & $V, G, R$ & F,C,Méd \\
\hline Albizzia lebbek & Z & A & $\mathrm{JFe}$ & $\mathrm{Bo}, \mathrm{Ca}, \mathrm{Ov}$ & SS & M & $\mathrm{G}, \mathrm{R}$ & $\mathrm{F}, \mathrm{C}$ \\
\hline Albizzia petersiana & $\mathrm{R}$ & $A$ & $\mathrm{Fe}, \mathrm{Et}$ & $\mathrm{Bo}, \mathrm{Ca}, \mathrm{Ov}$ & SS & - & - & - \\
\hline Dichrostachys cinerea & Z & a & $\mathrm{Fe}, \mathrm{Fr}, \mathrm{Go}$ & $\mathrm{Bo}, \mathrm{Ca}$ & SS,SP & $P$ à $T$ & $V, G, R$ & F,C,Méd \\
\hline Entada abyssinica & $\mathrm{B}, \mathrm{R}, \mathrm{Z}$ & a à $A$ & $\mathrm{Fe}, \mathrm{Et}$ & $\mathrm{Bo}, \mathrm{Ca}, \mathrm{Ov}$ & SS,SP & $P$ à $T$ & $V, G, R$ & F,C,Méd \\
\hline Entadopsis abyssinica & Z & $\mathrm{a}$ & $\mathrm{Fe}$ & Bo,Ca & SS,SP & M & $\mathrm{R}$ & F,C,Méd \\
\hline \multicolumn{9}{|l|}{ MORACEAE } \\
\hline Chlorophora excelsa & Z & A & $\mathrm{Fe}, \mathrm{Fr}$ & $\mathrm{Bo}, \mathrm{Ca}, \mathrm{Ov}$ & SS,SP & $\mathrm{T}$ & $\mathrm{V}, \mathrm{G}, \mathrm{R}$ & F,A,C,Méd \\
\hline Ficus brachylepis & $z$ & A & $\mathrm{Fe}, \mathrm{Et}$ & $\mathrm{Bo}, \mathrm{Ca}, \mathrm{Ov}$ & SS,SP & $\mathrm{T}$ & $\mathrm{V}, \mathrm{R}$ & F,C,D,Méd \\
\hline Ficus bubu & Z & A & $\mathrm{Fe}$ & $\mathrm{Bo}, \mathrm{Ca}, \mathrm{Ov}$ & SS,SP & $\mathrm{T}$ & $\mathrm{V}, \mathrm{R}$ & $\rightarrow$ \\
\hline Ficus capensis & Z & A & $\mathrm{Fr}$ & $\mathrm{Ca}$ & SP & $\mathrm{T}$ & $\mathrm{R}$ & F,Méd \\
\hline Ficus dekdekena & Z & A & $\mathrm{Fe}, \mathrm{Fr} \mathrm{sec}$ & $\mathrm{Bo}, \mathrm{Ca}, \mathrm{Ov}$ & SS,SP & $T$ & $\mathrm{~V}, \mathrm{G}, \mathrm{R}$ & $\mathrm{F}, \mathrm{C}$ \\
\hline Ficus exasperata & Z & A & $\mathrm{Fe}$ & $\mathrm{Bo}, \mathrm{Ca}, \mathrm{Ov}$ & SS,SP & $\mathrm{M}$ à $\mathrm{T}$ & $\mathrm{G}$ & $\mathrm{F}, \mathrm{D}$ \\
\hline Ficus glumosa & Z & A & $\mathrm{Fe}, \mathrm{Et}, \mathrm{Ec}$ & $\mathrm{Bo}, \mathrm{Ca}, \mathrm{Ov}$ & SS,SP & - & V & D,A,Méd \\
\hline Ficus gnaphalocarpa & Z & $A$ & $\mathrm{Fe}, \mathrm{Fr}$ & $\mathrm{Bo}, \mathrm{Ca}, \mathrm{Ov}$ & SS,SP & $P$ à $T$ & $V, G, R$ & F,C,Méd \\
\hline Ficus leprieurii & Z & A & $\mathrm{Fe}, \mathrm{Fr}$ & Bo,Rs & SS,SP & $M$ & $V, G, R$ & $\mathrm{~F}, \mathrm{C}$ \\
\hline Ficus natalensis & $\mathrm{R}$ & $a$ à $A$ & $\mathrm{Fe}$ & $\mathrm{Ca}, \mathrm{Ov}$ & SS & $\mathrm{T}(\mathrm{Ca})$ & $\mathrm{R}$ & $\mathrm{F}$ \\
\hline Ficus ovata & $\mathrm{R}, \mathrm{Z}$ & a à $A$ & $\mathrm{Fe}, \mathrm{Et}$ & $\mathrm{Bo}, \mathrm{Ca}, \mathrm{Ov}$ & SS,SP & $\mathrm{T}$ & $V, G, R$ & $\mathrm{~F}, \mathrm{D}, \mathrm{A}$ \\
\hline Ficus seretii & $\mathrm{Z}$ & A & $\mathrm{JFe}, \mathrm{Fr}$ & Bo & SS,SP & $\mathrm{T}$ & $V, G, R$ & F,C,Méd \\
\hline Ficus sp. (Kayimbi) $n^{\circ} 1$ & Z & A & $\mathrm{JFe}$ & $\mathrm{Bo}, \mathrm{Ca}, \mathrm{Ov}$ & SS,SP & $\mathrm{T}$ & $\mathrm{V}, \mathrm{R}$ & $F, C$ \\
\hline Ficus sp. (Kib) $n^{\circ} 2$ & $z$ & a & $\mathrm{Fe}$ & $\mathrm{Bo}, \mathrm{Ca}, \mathrm{Ov}$ & SS,SP & $\mathrm{T}$ & $\mathrm{V}, \mathrm{R}$ & $\mathrm{C}$ \\
\hline Ficus sp. (Lasong) $n^{\circ} 3$ & Z & $A$ & $\mathrm{Fe}$ & $\mathrm{Bo}, \mathrm{Ca}$ & SS,SP & $\mathrm{T}$ & $\mathrm{V}, \mathrm{R}$ & $\mathrm{C}$ \\
\hline Ficus sp. (Lekwen) $n^{\circ} 4$ & Z & A & $\mathrm{Fe}$ & $\mathrm{Ca}, \mathrm{Ov}$ & SS,SP & $\mathrm{T}$ & $\mathrm{V} ; \mathrm{R}$ & F,C,Méd \\
\hline Ficus sp. (Mutaba) $n^{\circ} 5$ & Z & $A$ & $\mathrm{Fe}$ & $\mathrm{Bo}, \mathrm{Ca}, \mathrm{Ov}$ & SS,SP & $\mathrm{T}$ & $\mathrm{V}, \mathrm{R}$ & $\mathrm{F}, \mathrm{C}$ \\
\hline Ficus sp. (Osuo) no 6 & Z & A & $\mathrm{Fe}, \mathrm{Ec}, \mathrm{Ca}$ & $\mathrm{Bo}, \mathrm{Ca}, \mathrm{Ov}$ & SS,SP & $\mathrm{T}$ & $\mathrm{V}, \mathrm{R}$ & $\mathrm{C}$ \\
\hline Ficus sp. $n^{\circ} 7$ & Z & $A$ & Fe,JPI & $\mathrm{Ca}, \mathrm{Ov}$ & SS,SP & $\mathrm{T}$ & $V, G, R$ & F,C,Méd \\
\hline Ficus thonningii & $\mathrm{B}, \overline{\mathrm{R}}, \mathrm{Z}$ & $A$ & $\mathrm{Fe}, \mathrm{Et}$ & $\mathrm{Bo}, \mathrm{Ca}, \mathrm{Ov}$ & SS,SP & - & $V, G, R$ & F,D,A,Méd \\
\hline Ficus vallis-choudae & $Z$ & A & $\mathrm{Fe}, \mathrm{Fr}$ & $\mathrm{Bo}, \mathrm{Ca}, \mathrm{Ov}$ & SS,SP & $\mathrm{T}(\mathrm{Fr})$ & $\mathrm{V}, \mathrm{G}$ & $\mathrm{F}$ \\
\hline Ficus vogeli & Z & $A$ & $\mathrm{Fe}, \mathrm{Et}$ & $\mathrm{Ca}, \mathrm{Ov}$ & SS,SP & - & V,R & F,D,A,Méd \\
\hline Musanga cecropioides & Z & $A$ & $\mathrm{Fe}$ & Bo & SS,SP & $M$ & $\mathrm{G}, \mathrm{R}$ & $\mathrm{F}, \mathrm{C}$ \\
\hline Myrianthus arboreus & Z & a à $A$ & $\mathrm{Fe}$ & $\mathrm{Ca}, \mathrm{OV}$ & SS,SP & $\mathrm{P}$ à $\mathrm{M}$ & $\mathrm{G}, \mathrm{R}$ & $\mathrm{F}$ \\
\hline \multicolumn{9}{|l|}{ MYRSINACEAE } \\
\hline Embelia schimperi & $\mathrm{B}, \mathrm{R}, \mathrm{Z}$ & $\mathrm{a}$ & $\mathrm{Fe}, \mathrm{Et}$ & $\mathrm{Bo}, \mathrm{Ca}, \mathrm{Ov}$ & SS,SP & - & - & Méd \\
\hline Maesa lanceolata & $\mathrm{B}, \mathrm{Z}$ & a à $A$ & $\mathrm{Fe}, \mathrm{Et}$ & $\mathrm{Bo}, \mathrm{Ca}, \mathrm{Ov}$ & SS,SP & $\mathrm{P}$ à $\mathrm{M}$ & $\mathrm{G}, \mathrm{R}$ & F,C,A,Méd \\
\hline \multicolumn{9}{|l|}{ MYRTACEAE } \\
\hline Syzygium cordatum & B & $\mathrm{a}$ à $\mathrm{A}$ & $\mathrm{Fe}, \mathrm{Et}$ & $\mathrm{Ca}, \mathrm{Ov}$ & SS & - & $R$ & $F, C$ \\
\hline Syzygium guineensis & Z & $\mathrm{a}$ à $\mathrm{A}$ & $\mathrm{Fe}$ & $\mathrm{Bo}, \mathrm{Ca}, \mathrm{Ov}$ & SS,SP & M & $\mathrm{V}, \mathrm{G}, \mathrm{R}$ & F,D,Méd \\
\hline \multicolumn{9}{|l|}{ OLACACEAE } \\
\hline Xymenia caffra & $\mathrm{R}$ & a & $\mathrm{Fe}, \mathrm{Fr}$ & $\mathrm{Bo}, \mathrm{Ca}, \mathrm{Ov}$ & SS,SP & 一 & 一 & 一 \\
\hline \multicolumn{9}{|l|}{ OLEACEAE } \\
\hline Olea africana & Z & a à $\mathrm{A}$ & $\mathrm{Fe}, \mathrm{Ec}$ & $\mathrm{Ca}, \mathrm{Ov}$ & SS,SP & - & V,R & F,C,Méd \\
\hline Schrebera alata & $Z$ & a à $\mathrm{A}$ & $\mathrm{Fe}$ & $\mathrm{Ca}$ & SS & $\mathrm{T}$ & $G, R$ & $F, C$ \\
\hline
\end{tabular}




\begin{tabular}{|c|c|c|c|c|c|c|c|c|}
\hline $\begin{array}{l}\text { FAMILLE } \\
\text { Espèces }\end{array}$ & Pays & Port & $\begin{array}{l}\text { Organes } \\
\text { appétés }\end{array}$ & $\begin{array}{l}\text { Animaux } \\
\text { consomm. }\end{array}$ & Epoques & $\begin{array}{l}\text { Appéti- } \\
\text { bilité }\end{array}$ & $\begin{array}{l}\text { Multipli- } \\
\text { cation }\end{array}$ & $\begin{array}{l}\text { Autres } \\
\text { Usages }\end{array}$ \\
\hline \multicolumn{9}{|l|}{ PALMAE } \\
\hline Elaeis guineensis & $z$ & A & $\mathrm{Fe}, \mathrm{Fr}$ & $\mathrm{Bo}, \mathrm{Ca}, \mathrm{Ov}$ & SS,SP & $\mathrm{T}$ & G & $\mathrm{D}$ \\
\hline Hyphaene thebaica & $\bar{Z}$ & $A$ & $\mathrm{Fr}$ & Bo & SS,SP & $\mathrm{T}$ & $\mathrm{G}$ & $A$ \\
\hline \multicolumn{9}{|l|}{ PIPERACEAE } \\
\hline Piper capense & $\mathrm{R}$ & s & $\mathrm{Fe}, \mathrm{Et}$ & Bo & SS,SP & - & - & - \\
\hline \multicolumn{9}{|l|}{ PITTOSPORACEAE } \\
\hline Pittosporum spathicalyx & $\mathrm{R}, \mathrm{Z}$ & a & $\mathrm{Fe}, \mathrm{Et}$ & $\mathrm{Bo}, \mathrm{Ca}, \mathrm{Ov}$ & SS,SP & - & $\mathbf{R}$ & $F, C$ \\
\hline \multicolumn{9}{|l|}{ POACEAE } \\
\hline Arundinaria alpina & $B, R, Z$ & Bambou & $\mathrm{Fe}, \mathrm{JPl}$ & $\mathrm{Bo}, \mathrm{Ca}, \mathrm{Ov}$ & SS,SP & - & V,R & $\mathrm{C}$ \\
\hline Bambusa vulgaris & Z & Bambou & JFe,JPI & $\mathrm{Bo}, \mathrm{Ca}$ & SS,SP & $\mathrm{T}$ & $\mathrm{V}, \mathrm{R}$ & $\mathrm{F}, \mathrm{C}$ \\
\hline Oxythenanthera abyssinica & $B, Z$ & Bambou & $\mathrm{Fe}, \mathrm{JPI}$ & $\mathrm{Bo}, \mathrm{Ca}, \mathrm{Ov}$ & SS,SP & $\mathrm{T}$ & $\mathrm{V}, \mathrm{R}$ & C,A,Méd \\
\hline \multicolumn{9}{|l|}{ POLYGALACEAE } \\
\hline Polygala ruwenzoriensis & Z & $a$ & $\mathrm{Fe}, \mathrm{Et}, \mathrm{Fl}$ & $\mathrm{Bo}, \mathrm{Ca}, \mathrm{Ov}$ & SS,SP & - & - & - \\
\hline Securidaca longepedunculata & $\mathrm{B}, \mathrm{Z}$ & a à $\mathrm{A}$ & $\mathrm{Fe}, \mathrm{Et}$ & $\mathrm{Bo}, \mathrm{Ca}, \mathrm{Ov}$ & SS,SP & $\mathrm{P}$ à $\mathrm{T}$ & $\mathrm{G}, \mathrm{R}$ & F,C,Méd \\
\hline \multicolumn{9}{|l|}{ POLYGONACEAE } \\
\hline Rumex usambarensis & $B, Z$ & a & $\mathrm{Fe}, \mathrm{Et}$ & $\mathrm{Bo}, \mathrm{Ca}, \mathrm{Ov}$ & SS,SP & - & $\mathrm{R}$ & Méd \\
\hline \multicolumn{9}{|l|}{ PROTEACEAE } \\
\hline $\begin{array}{l}\text { Protea madiensis } \\
\text { RHAMNACEAE }\end{array}$ & \multicolumn{8}{|c|}{ RHAMNACEAE } \\
\hline Scutia myrtina & $\mathrm{R}$ & a & $\mathrm{Fe}, \mathrm{Et}$ & $\mathrm{Ca}, \mathrm{Ov}$ & SS,SP & - & 一 & - \\
\hline \multicolumn{9}{|l|}{ RHYZOPHORACEAE } \\
\hline \multicolumn{9}{|l|}{ ROSACEAE } \\
\hline Hagenia abyssinica & $\mathrm{R}, \mathrm{Z}$ & $A$ & $\mathrm{Fe}, \mathrm{Et}$ & $\mathrm{Bo}, \mathrm{Ca}, \mathrm{Ov}$ & SS,SP & - & $\mathrm{V}, \mathrm{R}$ & $\begin{array}{c}A(Z), \\
\operatorname{Méd}(R)\end{array}$ \\
\hline \multicolumn{9}{|l|}{$R U B I A C E A E$} \\
\hline Canthium crassum & Z & $a$ & $\mathrm{Fe}$ & $\mathrm{Bo}, \mathrm{Ca}, \mathrm{Ov}$ & $\mathrm{SP}$ & $\mathrm{T}$ & $V, G, R$ & F,Méd \\
\hline Canthium guenzii & $\bar{Z}$ & $A$ & $\mathrm{JFe}, \mathrm{Et}, \mathrm{Fr}$ & $\mathrm{Bo}, \mathrm{Ca}, \mathrm{Ov}, \mathrm{Rs}$ & SS,SP & $M$ & $\mathrm{G}, \mathrm{R}$ & $F, C$ \\
\hline Cantium venosum & $\mathrm{B}$ & $b$ & $\mathrm{Fe}$ & $\mathrm{Bo}, \mathrm{Ca}, \mathrm{Ov}$ & SS,SP & - & - & - \\
\hline Craterispermum laurinum & Z & $a b$ & $\mathrm{JFe}$ & $\mathrm{Bo}, \mathrm{Ca}, \mathrm{Ov}$ & SS,SP & $\mathrm{T}$ & $V, G$ & F,C,Méd \\
\hline Cremaspora sp. & $Z$ & a & $\mathrm{JFe}$ & $\mathrm{Bo}, \mathrm{Ca}, \mathrm{Ov}, \mathrm{Rs}$ & SS,SP & $\mathrm{T}$ & $\mathrm{V}, \mathrm{G}, \mathrm{R}$ & F,C,Méd \\
\hline Crossopteryx febrifuga & Z & a à $A$ & $\mathrm{JFe}, \mathrm{Ec}$ & $\mathrm{Bo}, \mathrm{Ca}, \mathrm{Ov}$ & SS,SP & $P$ à $T$ & $\mathrm{G}, \mathrm{R}$ & F,C,A,Méd \\
\hline Gaertnena paniculata & $\bar{Z}$ & $\mathrm{a}$ & Ec & $\mathrm{Ca}$ & SS,SP & $\mathrm{T}$ & $G, R$ & $\mathrm{~F}$ \\
\hline Galiniera coffeoides & $B, Z$ & $\mathrm{a}$ & $\mathrm{Fe}, \mathrm{Et}$ & $\mathrm{Bo}, \mathrm{Ca}, \mathrm{Ov}$ & SS,SP & - & $R$ & $\mathrm{~F}$ \\
\hline Gardenia jovis-tonantis & Z & $a$ & $\mathrm{Fe}$ & Bo & SS,SP & $\mathrm{T}$ & $\mathrm{R}$ & F,Méd \\
\hline Gardenia ternifolia & $\overline{\mathrm{R}}$ & a & $\mathrm{Fe}, \mathrm{Et}, \mathrm{Fr}$ & $\mathrm{Bo}(\mathrm{Fr}), \mathrm{Ca}, \mathrm{Ov}$ & SS,SP & - & - & - \\
\hline Hymenodictyon floribundum & Z & $a$ & $\mathrm{Fe}, \mathrm{Et}, \mathrm{Ec}$ & $\mathrm{Ca}$ & SS,SP & - & $\mathrm{V}, \mathrm{R}$ & $\mathrm{F}, \mathrm{C}$ \\
\hline Leptactinia leopoldii II & $\bar{Z}$ & $a$ & $\mathrm{JFe}, \mathrm{Fr}$ & $\mathrm{Ca}$ & SS,SP & $M$ & $\mathrm{G}$ & - \\
\hline Morinda lucida & Z & A & $\mathrm{JFe}$ & $\mathrm{Bo}, \mathrm{Ca}, \mathrm{Ov}$ & SS,SP & $M$ & $V, G, R$ & F,C,Méd \\
\hline Mussaenda arcuata & $\mathbf{Z}$ & a & $\mathrm{JFe}$ & $\mathrm{BO}, \mathrm{Ca}, \mathrm{Ov}, \mathrm{Rs}$ & SS & $\mathrm{T}$ & $G, R$ & D \\
\hline Mitragyna rubrostipulosa & $\bar{Z}$ & $A$ & $\mathrm{Fe}, \mathrm{Et}$ & $\mathrm{Bo}, \mathrm{Ca}, \mathrm{Ov}$ & SS,SP & - & $\mathrm{V}, \mathrm{R}$ & $\mathrm{C}, \mathrm{A}$ \\
\hline Nauclea latifolia & $\bar{Z}$ & $a$ & $\mathrm{JFe}, \mathrm{Fr}$ & Bo & SS,SP & $T$ & $V, G, R$ & F,A,Méd \\
\hline Pavetta ternifolia & $B, R$ & a & $\mathrm{Fe}, \mathrm{Et}$ & $\mathrm{Bo}(\mathrm{B}), \mathrm{Ca}, \mathrm{Ov}$ & SS,SP & - & $\mathrm{V}, \mathrm{R}$ & $\mathrm{F}, \mathrm{C}$ \\
\hline \multicolumn{9}{|l|}{ Pseudosabicea arborea } \\
\hline Psychotria mahonii & R & a ou $A$ & $\mathrm{Fe}, \mathrm{Et}$ & Ca, Ov & SS,SP & - & - & - \\
\hline Vangueriopsis lanciflora & Z & $A$ & $\mathrm{JFe}, \mathrm{Fr}$ & $\mathrm{Bo}, \mathrm{Ca}, \mathrm{Ov}, \mathrm{Rs}$ & SS,SP & $M$ à T & $G, R$ & $\mathrm{~F}, \mathrm{C}$ \\
\hline Virectaria major & $B, R$ & $\mathrm{~b}$ & $\mathrm{Fe}, \mathrm{Et}$ & $\mathrm{Bo}, \mathrm{Ca}, \mathrm{Ov}$ & SS,SP & $\mathrm{T}(\mathrm{Ca})$ & $\mathrm{R}$ & F,Méd \\
\hline \multicolumn{9}{|l|}{ RUTACEAE } \\
\hline Clausena anisata & $z$ & a à $A$ & $\mathrm{Fe}, \mathrm{Et}, \mathrm{Ec}$ & $\mathrm{Bo}, \mathrm{Ca}, \mathrm{Ov}$ & SS,SP & $P$ à $T$ & $G, R$ & F,Méd \\
\hline SAPINDACEAE & & & & & & & & \\
\hline Allophyllus africanus & $\mathrm{R}$ & a à $A$ & $\mathrm{Fe}$ & $\mathrm{Bo}, \mathrm{Ca}, \mathrm{Ov}$ & sS & - & - & - \\
\hline
\end{tabular}


TABLEAU I (suite)

\begin{tabular}{|c|c|c|c|c|c|c|c|c|}
\hline $\begin{array}{l}\text { FAMILLE } \\
\text { Espèces }\end{array}$ & Pays & Port & $\begin{array}{l}\text { Organes } \\
\text { appétés }\end{array}$ & $\begin{array}{c}\text { Animaux } \\
\text { consomm. }\end{array}$ & Epoques & $\begin{array}{l}\text { Appéti- } \\
\text { bilité }\end{array}$ & $\begin{array}{l}\text { Multipli- } \\
\text { cation }\end{array}$ & $\begin{array}{c}\text { Autres } \\
\text { Usages }\end{array}$ \\
\hline Allophyllus rubifolius & $\mathrm{R}$ & a & $\mathrm{Fe}, \mathrm{Et}$ & $\mathrm{Ca}, \mathrm{Ov}$ & SS,SP & 一 & $\mathrm{R}$ & - \\
\hline Dodonea viscosa & $B, R, Z$ & a & $\mathrm{Fe}, \mathrm{Et}$ & $\mathrm{Bo}, \mathrm{Ca}, \mathrm{Ov}$ & SS,SP & 一 & $R$ & F,C,Méd \\
\hline SOLANACEAE & & & & & & & & \\
\hline Solanum aculeastrum & $B, R, Z$ & a & $\mathrm{Fe}, \mathrm{Et}, \mathrm{Fr}$ & $\mathrm{Ca}, \mathrm{Ov}$ & SS,SP & 一 & $\mathrm{R}$ & $F$ \\
\hline Solanum dasyphyllum & Z & a & $\mathrm{JFe}$ & $\mathrm{Bo}, \mathrm{Ca}, \mathrm{Ov}$ & SS & M & $\mathrm{G}, \mathrm{R}$ & $F$ \\
\hline Solanum incanum & $\bar{Z}$ & a & $\mathrm{Fe}, \mathrm{Et}$ & $\mathrm{Ca}, \mathrm{Ov}$ & SS,SP & M & $\mathrm{G}$ & - \\
\hline STERCULIACEAE & & & & & & & & \\
\hline Dombeya bagshawei & $\mathrm{B}, \mathrm{R}, \mathrm{Z}$ & $\mathrm{a}$ à $\mathrm{A}$ & $\mathrm{Fe}, \mathrm{Et}$ & $\mathrm{Bo}, \mathrm{Ca}, \mathrm{Ov}$ & SS,SP & $M$ à $T$ & $\mathrm{G}, \mathrm{R}$ & F,C,Méd \\
\hline Dombeya goetzenii & Z & a à $A$ & $\mathrm{Fe}, \mathrm{Et}$ & $\mathrm{Bo}, \mathrm{Ca}, \mathrm{Ov}$ & SS,SP & - & $\mathrm{G}, \mathrm{R}$ & $F, D$ \\
\hline Dombeya rotundifolia & $\bar{Z}$ & $\mathrm{a}$ & $\mathrm{Fe}, \mathrm{Fr}$ & $\mathrm{BO}, \mathrm{Ca}$ & SS & $\mathrm{T}(\mathrm{Fr}) \mathrm{P}(\mathrm{Fe})$ & $V, G$ & F,Méd \\
\hline TILIACEAE & & & & & & & & \\
\hline Grewia cf. bicolor & $B$ & $a b$ & $\mathrm{Fe}$ & $\mathrm{Bo}, \mathrm{Ca}$ & SS & - & - & - \\
\hline Grewia forbesii & $z$ & $a$ & $\mathrm{Fe}, \mathrm{Fr}$ & $\mathrm{Bo}, \mathrm{Ca}, \mathrm{Ov}$ & SS,SP & - & - & - \\
\hline Grewia mollis & $\bar{Z}$ & a & $\mathrm{Fe}, \mathrm{Et}$ & $\mathrm{Bo}, \mathrm{Ca}, \mathrm{Ov}$ & SS & $\mathrm{T}$ & $G, R$ & F,C,A,Méd \\
\hline Grewia platyclada & $\mathrm{B}, \mathrm{Z}$ & a & $\mathrm{Fe}, \mathrm{Et}$ & $\mathrm{Bo}, \mathrm{Ca}, \mathrm{Ov}$ & SS & - & - & D \\
\hline Grewia pubescens & $\mathrm{B}$ & a ou $A$ & $\mathrm{Fe}$ & $\mathrm{Bo}, \mathrm{Ca}$ & SS,SP & $\mathrm{T}(\mathrm{SS})$ & 一 & - \\
\hline Grewia trichocarpa & $\mathrm{R}$ & a à $A$ & $\mathrm{Fe}, \mathrm{Et}$ & $\mathrm{Bo}, \mathrm{Ca}, \mathrm{Ov}$ & SS,SP & - & 一 & - \\
\hline Triumfetta cordifolia & $B, Z$ & a & $\mathrm{Fe}, \mathrm{Et}$ & $\mathrm{Bo}, \mathrm{Ca}, \mathrm{Ov}$ & SS,SP & $\mathrm{T}(\mathrm{Ca}, \mathrm{SS})$ & $\mathrm{R}$ & F,D,Méd \\
\hline Triumfetta sp. & Z & a & $\mathrm{Fe}, \mathrm{Et}$ & $\mathrm{Bo}, \mathrm{Ca}, \mathrm{Ov}$ & SS & $\mathrm{T}$ & $\mathrm{G}, \mathrm{R}$ & $\mathrm{F}, \mathrm{C}, \mathrm{A}$ \\
\hline ULMACEAE & & & & & & & & \\
\hline Celtis africana & Z & A & $\mathrm{Fe}, \mathrm{Et}, \mathrm{Ec}$ & $\mathrm{Bo}, \mathrm{Ca}, \mathrm{Ov}$ & SS,SP & 一 & $G$ & - \\
\hline Trema orientalis & $\mathrm{B}, \mathrm{Z}$ & a à $A$ & $\mathrm{Fe}, \mathrm{Et}$ & $\mathrm{Bo}, \mathrm{Ca}, \mathrm{Ov}$ & SS,SP & 一 & $\mathrm{R}$ & $F, C$ \\
\hline VERBENACEAE & & & & & & & & \\
\hline Clerodendrum buchholzii & $\mathrm{R}$ & a & $\mathrm{Fe}, \mathrm{Et}$ & $\mathrm{Ca}, \mathrm{OV}$ & SS,SP & - & - & - \\
\hline Clerodendrum myricoides & $\mathrm{R}$ & $b, a, A$ & $\mathrm{Fe}, \mathrm{Et}, \mathrm{Fr}$ & $\mathrm{Bo}, \mathrm{Ca}, \mathrm{Ov}$ & SS,SP & $\mathrm{T}$ (veaux) & - & - \\
\hline Clerodendrum rotundifolium & $\mathrm{R}, \mathrm{Z}$ & $\mathrm{b}$ & $\mathrm{Fe}, \mathrm{Et}$ & $\mathrm{Bo}, \mathrm{Ca}, \mathrm{Ov}$ & SS,SP & $M$ & - & Méd \\
\hline Clerodendrum schweinfurthii & $\mathrm{B}$ & $a b$ & $\mathrm{Fe}, \mathrm{Et}$ & $\mathrm{Ca}$ & SS,SP & 一 & - & - \\
\hline Lantana trifolia & $\mathrm{R}$ & $a b$ & $\mathrm{Fe}, \mathrm{Et}, \mathrm{Fl}, \mathrm{Fr}$ & $\mathrm{Bo}, \mathrm{Ca}, \mathrm{Ov}$ & SS,SP & - & - & - \\
\hline Lippia multiflora & Z & $\mathrm{a}$ & $\mathrm{JFe}$ & $\mathrm{Bo}, \mathrm{Ca}, \mathrm{Ov}$ & SP,SS & $\mathrm{T}$ & $V, G, R$ & Méd \\
\hline Vitex doniana & $\mathrm{B}, \mathrm{Z}$ & $a$ à $A$ & $\mathrm{~F} \theta, \mathrm{Et}$ & $\mathrm{Bo}, \mathrm{Ca}, \mathrm{Ov}$ & SS & $M$ à $T$ & $\mathrm{G}, \mathrm{R}$ & F,Méd \\
\hline Vitex ferruginea & Z & $A$ & $\mathrm{JFe}$ & $\mathrm{Bo}, \mathrm{Ca}, \mathrm{Ov}$ & SP,SS & $P$ à $T$ & $\mathrm{G}, \mathrm{R}$ & F,A,C,Méd \\
\hline Vitex fischerii & $\mathrm{Z}$ & a & $\mathrm{Fe}, \mathrm{Et}$ & $\mathrm{Ca}, \mathrm{Ov}$ & SP & $M$ & $\mathrm{~V}, \mathrm{R}$ & F,C \\
\hline Vitex madiensis & $Z$ & a & $\mathrm{JFe}, \mathrm{Et}, \mathrm{Fr}$ & $\mathrm{Bo}, \mathrm{Ca}, \mathrm{Ov}, \mathrm{Rs}$ & SP,SS & $P$ à $T$ & $\mathrm{G}, \mathrm{R}$ & F,C,Méd \\
\hline Vitex mombassae & $\bar{Z}$ & A & $\mathrm{JFe}, \mathrm{Fr}, \mathrm{Ec}$ & $\mathrm{Bo}, \mathrm{Ca}, \mathrm{Ov}, \mathrm{Rs}$ & SS,SP & M à T & $G, V, R$ & F,C,D,Méd \\
\hline
\end{tabular}

Pays : $B=$ Burundi $;=$ Rwanda $; Z=$ Zaïre.

Port: $A=$ arbre $; a=$ arbuste $; a b=$ arbrisseau $; b=$ buisson $; s=$ suffrutex.

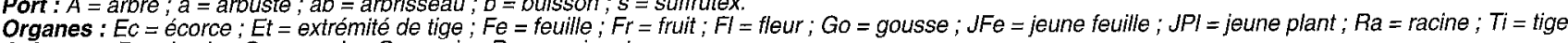
Animaux : $B o=$ bovin $; C a=$ caprin; $O V=o v i n ; R s=$ ruminant sauvage

Epoques : $S S=$ saison sèche; $S P=$ saison des pluies.

Degré d'appétibilité $: M=$ moyen $; P=$ peu; $T=$ très.
Multiplication : $G=$ générative $V-$ végétative $P=$ rejet.

Autres usages : $A=$ artisanal $; C=$ construction $; D=$ domestique $; F=$ feu $;$ Méd $=$ médicinal $; O=$ ornemental

\section{Pouvoir de rejeter}

Dans la perspective de l'utilisation rationnelle des arbres, arbrisseaux et arbustes fourragers, le fait qu'une espèce rejette après coupe permet une exploitation plus longue sans devoir la replanter plusieurs fois. Cet aspect a intéressé les différentes prospections et la grande majorité des espèces $(69 p .100)$ rejette après coupe.

\section{Autres usages}

Les espèces à usages multiples devant être priviligiées dans les actions d'agroforesteric, les prospections se sont intéressées aussi aux usages autres que fourragers. Pour l'éleveur, les principaux rôles reconnus sont ceux de tout arbre, c'est-à-dire son bois pour le feu (60,6 p. 100 des espèces) et les constructions diverses (43,3 p. 100) ainsi que son rôle médicinal (42,6 p. 100) dévolu surtout aux feuilles, racines et écorces et, parfois, son rôle dans l'alimentation humaine et l'économie domestique.

\section{Analyses chimiques}

Des analyses chimiques des feuilles ont été effectuées sur 24 espèces communes au Burundi, au Rwanda et aux régions du Nord et du Sud-Kivu récoltées au Burundi en saison des pluies (mars-avril) et en saison sèche (août). Les résultats figurent au tableau II.

On peut constater que la teneur en matières azotées des espèces analysées varie entre 12 et 31 p. 100 de la matière sèche pour une moyenne de 18,5 p. 100 en saison des pluies et entre 9 et 30 p. 100 de la matière sèche pour une moyenne de 17,6 p. 100 en saison sèche. 
TABLEAU II Analyse chimique de 24 espèces communes pour le Burundi, le Rwanda et le Zaïre (en p. 100 MS).

\begin{tabular}{|c|c|c|c|c|c|c|c|c|c|c|}
\hline \multirow{2}{*}{ Espèces } & \multicolumn{2}{|c|}{ MAT } & \multicolumn{2}{|c|}{ CELL } & \multicolumn{2}{|c|}{ Cendres } & \multicolumn{2}{|c|}{$\mathrm{Ca}$} & \multicolumn{2}{|c|}{$\mathrm{P}$} \\
\hline & $\mathrm{SP}$ & SS & SP & SS & $\mathrm{SP}$ & SS & $\mathrm{SP}$ & SS & SP & SS \\
\hline Acanthus pubescens & 18,7 & 23,0 & 10,2 & 36,0 & 10,6 & 10,3 & 0,82 & 1,44 & 0,29 & 0,27 \\
\hline Brillantaisia cicatricosa & 23,7 & 22,9 & 16,3 & 33,2 & 11,3 & 10,6 & 2,34 & 1,63 & 0,17 & 0,16 \\
\hline Dracena afromontana & 17,2 & 19,5 & 28,1 & 40,8 & 11,0 & 10,2 & 1,20 & 1,10 & 0,19 & 0,21 \\
\hline Rhus natalensis & 17,3 & 14,1 & 16,5 & 28,5 & 4,5 & 5,8 & 0,42 & 0,78 & 0,23 & 0,18 \\
\hline Rhus vulgaris & 13,5 & 11,4 & 14,7 & 32,9 & 5,1 & 5,6 & 0,53 & 0,57 & 0,17 & 0,12 \\
\hline Senecio mannii & 21,5 & 21,9 & 13,2 & 31,9 & 16,9 & 13,0 & 1,33 & 1,18 & 0,30 & 0,23 \\
\hline Vernonia amygdalina & 24,0 & 23,9 & 14,0 & 28,9 & 10,1 & 10,3 & 1,01 & 0,68 & 0,26 & 0,25 \\
\hline Parinari curatellifolia & 15,8 & 12,9 & 26,4 & 44,9 & 5,6 & 5,6 & 0,41 & 0,26 & 0,14 & 0,18 \\
\hline Harungana madagascariensis & 11,9 & 9,9 & 15,5 & 30,4 & 5,1 & 3,8 & 0,76 & 0,61 & 0,28 & 0,18 \\
\hline Euphorbia tirucalli & - & 8,9 & 31,8 & 53,7 & 11,1 & 12,1 & 1,64 & 1,56 & 0,23 & 0,18 \\
\hline Synadenium grantii & 13,1 & 11,6 & 17,8 & 30,7 & 14,3 & 14,9 & 1,39 & 1,49 & 0,23 & 0,30 \\
\hline Erythrina abyssinica & 19,8 & 20,3 & 31,9 & 53,3 & 6,2 & 7,3 & - & 0,67 & 0,26 & 0,19 \\
\hline Acacia gerrardi & 14,0 & 24,3 & 12,9 & 40,8 & 4,1 & 6,5 & 0,89 & 1,20 & 0,15 & 0,16 \\
\hline Acacia hockii & 31,1 & 13,7 & 17,8 & 25,8 & 6,6 & 5,7 & 1,69 & 1,13 & 0,15 & 0,13 \\
\hline Albizzia adianthifolia & - & 29,9 & 28,8 & 26,0 & 4,4 & 4,3 & 0,22 & 0,22 & 0,30 & 0,33 \\
\hline Entada abyssinica & 22,8 & 18,3 & 19,3 & 27,1 & 3,9 & 4,1 & 0,81 & 0,35 & 0,16 & 0,18 \\
\hline Ficus gnaphalocarpa & 11,6 & 15,7 & 17,9 & 33,7 & 8,0 & 9,2 & 0,93 & 0,59 & 0,14 & 0,14 \\
\hline Embelia schimperi & 15,0 & 14,1 & 16,6 & 32,2 & 4,4 & 4,1 & 0,81 & 0,72 & 0,24 & 0,16 \\
\hline Arundinaria alpina & 18,6 & 26,2 & 25,1 & 27,0 & 14,7 & 12,1 & 0,30 & 0,32 & 0,17 & 0,16 \\
\hline Pavetta ternifolia & 16,2 & 15,3 & 15,2 & 28,9 & 4,0 & 4,7 & 0,45 & 0,72 & 0,19 & 0,16 \\
\hline Dodonea viscosa & 15,1 & 11,9 & 15,7 & 43,9 & 4,8 & 3,2 & 0,45 & 0,36 & 0,20 & 0,12 \\
\hline Solanum aculeastrum & 24,2 & 22,6 & 20,1 & 35,7 & 10,6 & 7,9 & 1,38 & 1,15 & 0,32 & 0,17 \\
\hline Dombeya bagshawei & 18,9 & 19,2 & 16,8 & 34,5 & 9,0 & 8,2 & 1,65 & 1,33 & 0,23 & 0,19 \\
\hline Grewia platyclada & 22,2 & 17,4 & 18,2 & 37,9 & 9,2 & 8,3 & 1,45 & 1,42 & 0,30 & 0,21 \\
\hline
\end{tabular}

Source : Laboratoire de chimie agricole de l'institut des sciences agronomiques du Burundi (ISABU).

$M S=$ matière sèche $; M A T=$ matières azotées totales $; C E L L=$ cellulose de Kurschner $; S P=$ saison des pluies; $S S=$ saison sèche.

\section{DISCUSSION}

Une centaine d'espèces inventoriées dans ce document ont déjà été citées dans les communications présentées au Colloque sur les fourrages ligneux en Afrique (3).

L'appartenance d'une espèce à une liste de prospection tient au fait qu'elle a été signalée comme broutée. Son absence ne signifie donc nullement qu'elle n'y existe pas comme espèce botanique.

La différence entre les feuilles et les extrémités de tiges n'est pas nette car, dans la plupart des cas, l'animal qui recherche en priorité des jeunes feuilles consomme en même temps les extrémités des tiges tendres.

L'importance du brout par les animaux dépend de l'espèce animale, ce qui rejoint l'affirmation de SKERMAN (6) selon laquelle : "le mouton se nourrit surtout des graminées, des plantes herbacées et des plantes broutables basses; le régime des bovins est à base de graminées mais complété par du brout et des gousses ; quant aux caprins, leur régime est à base de brout et de gousses, complété par des graminées". Dans la région, il est de coutume de faire pâturer les ovins et les bovins ensemble et les caprins séparément. II convient cependant d'ajouter à ce qui précède que la saison, la disponibilité en graminées, l'accoutumance et la densité des ligneux influent sur le recours au brout.
III est important de préciser, en outre, que le fait qu'une ou deux espèces animales soient désignées comme consommatrices peut également signifier que seule(s) cette (ou ces) espèce est (sont) élevée(s) dans la région prospectée.

L'intensité du brout, un peu plus prononcée en saison sèche qu'en saison des pluies, est due à deux raisons :

- les arbres et arbustes, par leur enracinement profond, restent verts pendant la saison sèche alors que les graminées sèchent. De plus, les arbres et arbustes émettent de jeunes feuilles avant le retour des pluies ;

- dans la plupart des régions prospectées, les pâturages sont souvent brûlés en milieu de saison sèche pour provoquer la repousse des graminées. Pendant que les herbes sont encore basses, les arbres émettent de jeunes feuilles très tendres qui incitent au brout.

Quant aux usages autres que fourragers, on retrouve les mêmes qu'ailleurs en Afrique $(5,7)$.

Les teneurs en matières azotées, en moyenne 18,5 p. 100 de la matière sèche, des quelques espèces analysées sont supérieures à celle rapportées par divers auteurs pour l'Afrique de l'Ouest et de l'Afrique de l'Est: respectivement 12,5 et 13,3 p. 100 de la matières sèche $(2,4)$ 


\section{CONCLUSION}

Les prospections ont recensé, dans la flore naturelle de la zone, des espèces de ligneux dont certains organes sont consommés par les bovins, les caprins et les ovins et qui se répartissent dans des familles botaniques fort diversifiées.

L'analyse chimique de quelques espèces a donné une idée sur la bonne valeur bromatologique et sur la faible variabilité de la teneur en matière azotée totale au cours de l'année. L'éleveur traditionnel a une bonne connaissance des arbres et arbustes fourragers indigènes. Cette connaissance est cependant passive. Elle est surtout basée sur une observation de ce que les animaux prélèvent au pâturage sans qu'elle ne conduise ni à une distribution par l'éleveur des organes appétés, ni à une gestion quelconque des essences broutées.

Toutes ces considérations indiquent qu'il y a là un champ immense de recherche dont l'objectif sera de déterminer, parmi les essences prospectées comme fourragères, celles présentant un réel intérêt dans l'alimentation des ruminants. Les espèces à usages multiples produisant de bons fourrages et pouvant jouer également le rôle d'arbres/arbustes agroforestiers dans les systèmes d'exploitation du paysan seront les plus recherchées.

\section{REMERCIEMENTS}

Ce travail a été réalisé dans le cadre des activités de l'auteur à l'Institut de Recherches agronomique et zootechnique (IRAZ) de la Communauté économique des Pays des Grands Lacs. Que son comité de gestion trouve ici l'expression de sa profonde gratitude.

L'auteur remercie particulièrement $M$. Michel BAUMER pour avoir assuré la correction et l'édition de cet article alors qu'il était chercheur principal à l'International Council for Research in Agroforestry.

MUZINGA KANZILA. Browse trees survey in the Communauté ćconomique des Pays des Grands Lacs (Burundi, Rwanda, Zaire). Revue Élev. Méd. vét. Pays trop., 1994, 47 (4): 415-424

In a survey of farmers and herdsmen conducted in areas of Burundi, Rwanda and Zaire with large livestock populations, 242 indigenous tree and shrub species from $\mathbf{5 3}$ families were cited as being used as fodder by cattle, goats and sheep. All the domesticated ruminants in the survey area consume fodder trees and shrubs at one time or another during the year, depending upon fodder availability and upon their own animal species. The period of greatest consumption was the dry season. Whereas the overall utility of the species was known, the farmers did not attach great importance to their fodder use and so did not managed them as such. The nitrogen content of some selected species was 18.5 and $17.6 \%$ of dry matter in samples collected during the rainy and dry seasons, respectively.

Key words : Cattle - Sheep - Goat - Browse plant - Feeding - Organoleptic property - Chemical composition - Season - Burundi - Rwanda - Zaire.

\section{BIBLIOGRAPHIE}

1. AUDRU (J). Les ligneux et subligneux fourragers et fruitiers en zone guinéenne : leurs perspectives d'emploi en élevage. In : LE HOUÉROU (H.N.), éd. Les fourrages ligneux en Afrique. Etat actuel des connaissances. Papiers présentés au Colloque sur les fourrages ligneux en Afrique, Addis Abeba, 8-12 avril 1980, et autres contributions. Addis Abeba, Ethiopie, CIPEA. p. 117-122.

2. LAMPREY (H.F.), HERLOCKER (D.J.), FIELD (C.R.). Les ligneux fourragers en $\Lambda$ frique de l'Est. In : LE HOUÉROU (H.N.), éd. Les fourrages ligneux en Afrique. Etat actuel des connaissances. Papiers présentés au Colloque sur les fourrages ligneux en Afrique, Addis Abeba, 8-12 avril 1980, el autres contributiuns. Adüis Abeba, Ethiopie, CIPEA. p. 33-55.

3. LE HOUÉROU (H.N.), éd. Les fourrages ligneux en Afrique. Etat actuel des connaissances. Papiers présentés au Colloque sur les fourrages ligneux en Afrique, Addis Abeba, 8-12 avril 1980, et autres contributions. Addis Abeba, Ethiopie, CIPEA, 1980. 481 p.

4. LE HOUÉROU (H.N.). Composition chimique et valeur nutritive des fourrages ligneux en Afrique tropicale occidentale. In : LE HOUÉROU (H.N.), éd. Les fourrages ligneux en Afrique. Etat actuel des connaissances. Papiers présentés au Colloque sur les fourrages ligneux en Afrique, Addis Abeba, 8-12 avril 1980, et autres contributions. Addis Abeba, Ethiopie, CIPEA. p. 259-284.

5. McKELL (C.M.). Utilisations multiples des arbres et arbustes fourragers : perspectives mondiales. In : LE HOUÉROU (H.N.), éd. Les fourrages ligneux en Afrique. Etat actuel des connaissances. Papiers présentés au Colloque sur les fourrages ligneux en Afrique, Addis Abeba, 8-12 avril 1980, et autres contributions. Addis Abeba, Ethiopie, CIPEA. p. 139-148.

\section{SKERMAN (P.J.). Tropical forage legumes. Rome, FAO, 1977.}

7. WICKENS (G.E.). Autres utilisations des espèces ligneuses. In : LE HOUÉROU (H.N.), éd. Les fourrages ligneux en Afrique. Etat actuel des connaissances. Papiers présentés au Colloque sur les fourrages ligneux en Afrique, Addis Abeba, 8-12 avril 1980, et autres contributions. Addis Abeba, Ethiopie, CIPEA. p. 153-180.

MUZINGA KANZILA. Estudios de las plantas leñosas forrajeras en el area de la Communauté économique des Pays des Grands Lacs (Burundi, Rwanda, Zaire). Revue Élev. Méd. vét. Pays trop., 1994, 47 (4) : 415-424

Los estudios realizados con los criadores y boyeros de las grandes regiones de crianza de Burundi, Rwanda y Zaire, permitieron hacer el inventario de' 242 especies de 'árboles y arbustos nativos, consumidos por los bovinos, caprinos y ovinos. Estas especies se repartieron en 53 familias botánicas. Todos los rumiantes domésticos de las regiones estudiadas pastorean en algún momento del año, según la disponibilidad de las hierbas forrajeras y sobre todo según la especie animal a la que pertenecen. La máxima época para la recolección de muestras es la estación seca. El productor tradicional tiene un buen conocimiento de los árboles y arbustos forrajeros nativos, sin embargo, la principal utilidad que les reconoce es la de cualquier árbol. El uso como forrajeros es el objeto de una simple observación, razón por la cual los árboles $\mathrm{y}$ arbustos forrajeros no reciben ningún trato particular. Es de 18,5 p. 100 y de 17,6 p. 100 respectivamente el promedio de materias nitrogenadas de la materia seca en algunas especies durante la estación lluviosa y la estación seca.

Palabras clave : Bovino - Caprino - Ovino - Planta de ramoneo - Alimentación - Propiedad organolcptica - Composición química - Estación - Burundi Rwanda - Zaire. 